\title{
Niobium Bis-Alkylidene Complexes Prepared by a Two- Electron Redox Process
}

Uriah Kilgore, John Tomaszewski, Hongjun Fan, John C. Huffman, Daniel J. Mindiola*

\section{Experimental Section.}

General Considerations. Unless otherwise stated, all operations were performed in a M. Braun Lab Master double-dry box under an atmosphere of purified nitrogen or using high vacuum standard Schlenk techniques under an argon atmosphere. Anhydrous solvents were dried according to literature procedures. ${ }^{1}$ Deutero arenes and alkanes were purchased from Cambridge Isotope Laboratory (CIL), degassed and vacuum transferred to $4 \AA$ molecular sieves. Celite, alumina, and $4 \AA$ molecular sieves were activated under vacuum overnight at $200{ }^{\circ} \mathrm{C}$. Complex 1 was prepared according to the literature. ${ }^{2}$ $\mathrm{ClMgCH}_{2} \mathrm{CMe}_{3}$ was purchased from Rieke Metals. $\mathrm{Mg}\left(\mathrm{CH}_{2} \mathrm{SiMe}_{3}\right)_{2}$ and $\mathrm{Mg}\left(\mathrm{CH}_{2} \mathrm{CMe}_{3}\right)_{2}$ (dioxane) were prepared according to the literature methods. ${ }^{3,4}$ $\mathrm{LiCH}_{2} \mathrm{SiMe}_{3}$ was purchased from Aldrich as a $1.0 \mathrm{M}$ solution in pentane. The solution was concentrated under reduced pressure then crystallized by storing at $-35{ }^{\circ} \mathrm{C}$. The white solid was collected, dried under reduced pressure, and stored at $-35^{\circ} \mathrm{C}$. All other chemicals were used as received. CHN analyses were performed by Midwest Microlabs, Indianapolis IN. ${ }^{1} \mathrm{H},{ }^{13} \mathrm{C},{ }^{29} \mathrm{Si}$ and ${ }^{31} \mathrm{P}$ NMR spectra were recorded on Varian 500,400 or $300 \mathrm{MHz}$ NMR spectrometers. ${ }^{1} \mathrm{H}$ and ${ }^{13} \mathrm{C}$ NMR are reported with reference to residual solvent resonances. ${ }^{31} \mathrm{P}$ NMR chemical shifts are reported with respect to external $\mathrm{H}_{3} \mathrm{PO}_{4}$ (0.0 ppm). ${ }^{29} \mathrm{Si}$ NMR shifts are reported with respect to external $\mathrm{SiMe}_{4}$ at $0 \mathrm{ppm}$. 


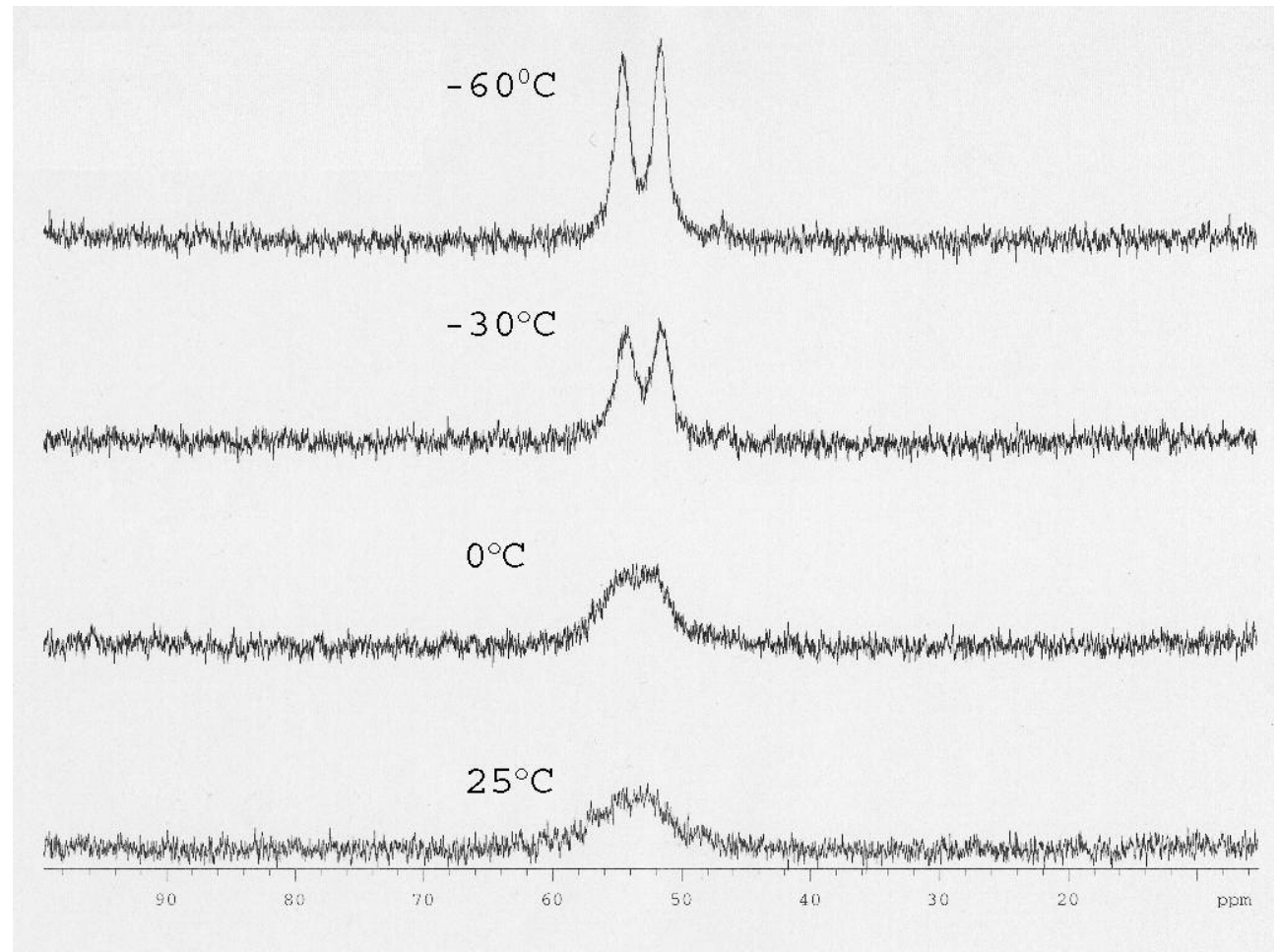

Figure S1. ${ }^{31} \mathrm{P}-\mathrm{NMR}$ spectra of $\mathbf{4}$ at various temperatures. 


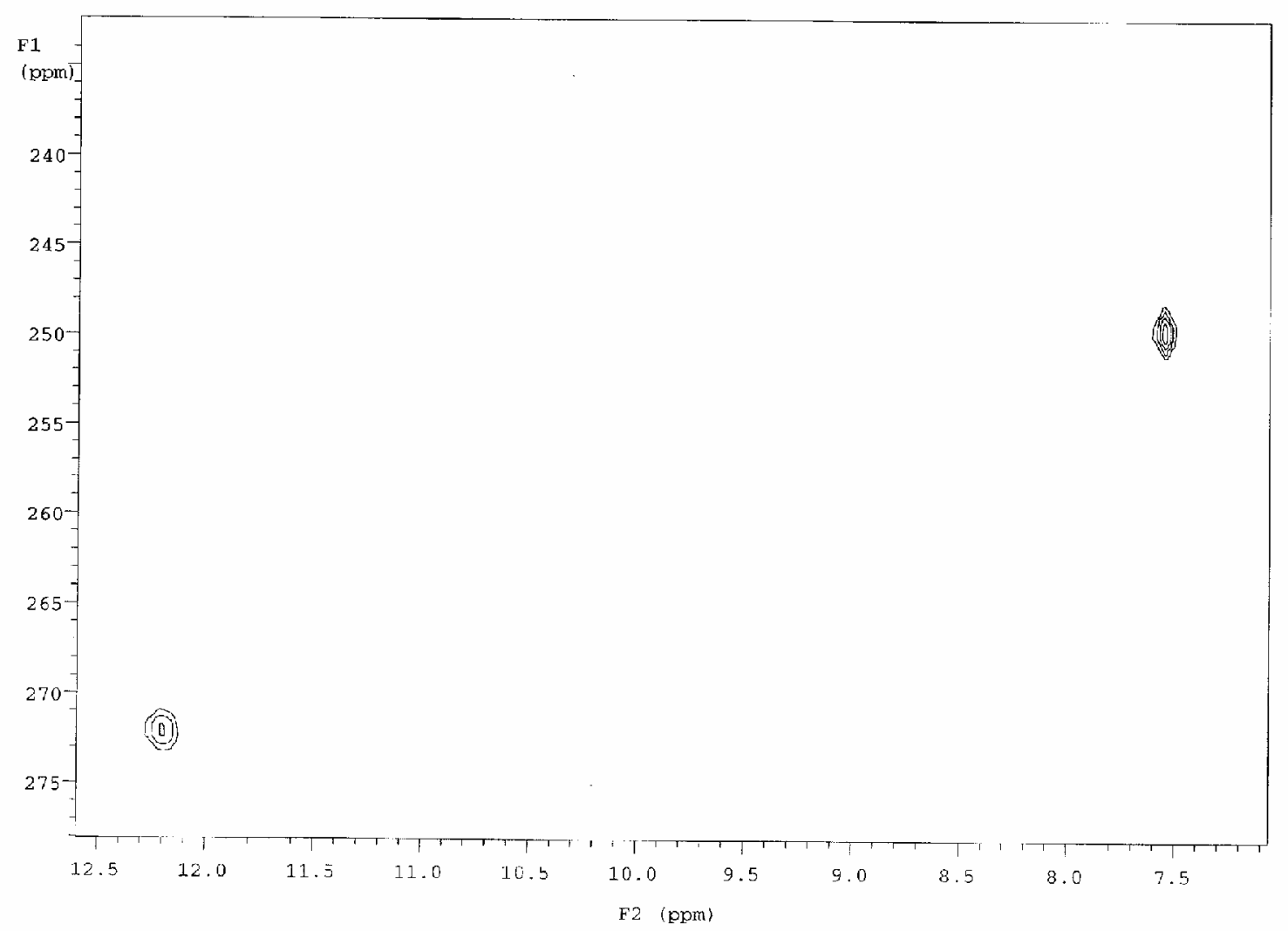

Figure S2. gHMQC spectrum of $\mathbf{4}$ showing correlations of $\alpha$-carbons to $\alpha$-hydrogens. 


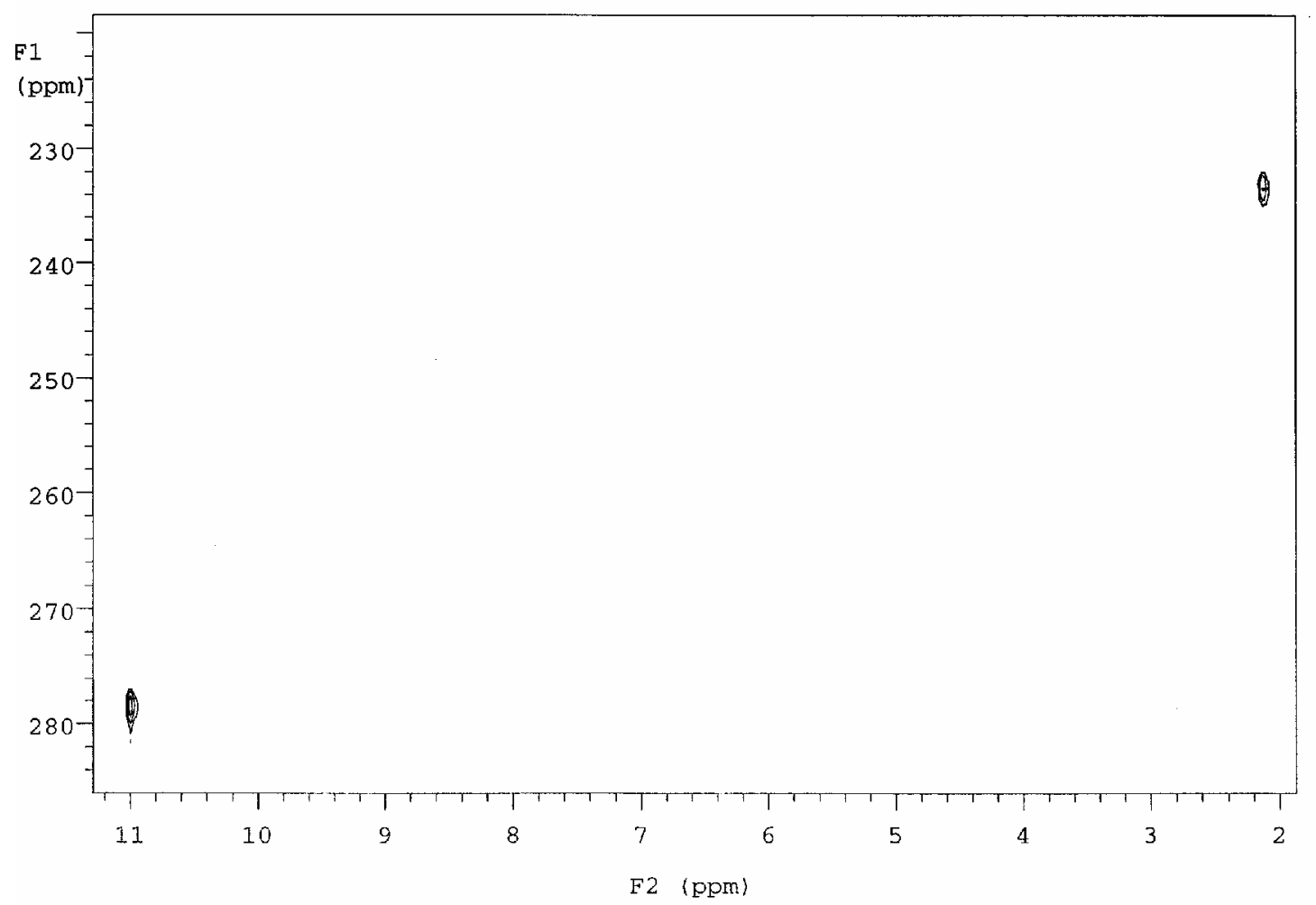

Figure S3. gHMQC spectrum of 5 showing correlations of $\alpha$-carbons to $\alpha$-hydrogen. 

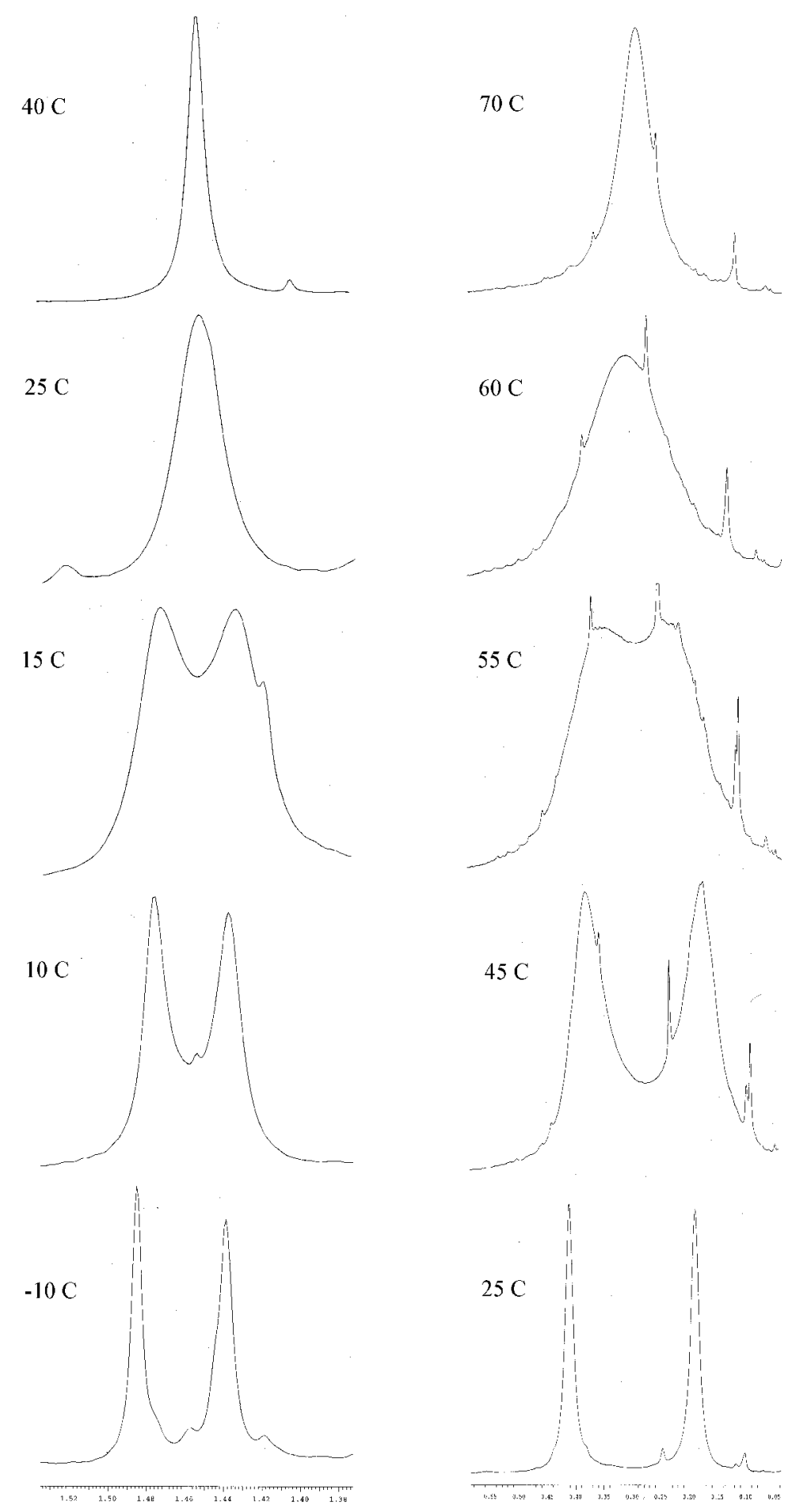
Figure S4. Variable temperature ${ }^{1} \mathrm{H}-\mathrm{NMR}$ spectrum of $\mathbf{4}$ (right) and $\mathbf{5}$ (left) focusing on $\mathrm{CMe}_{3}$ and $\mathrm{SiMe}_{3}$ resonances above and below coalescence temperatures.

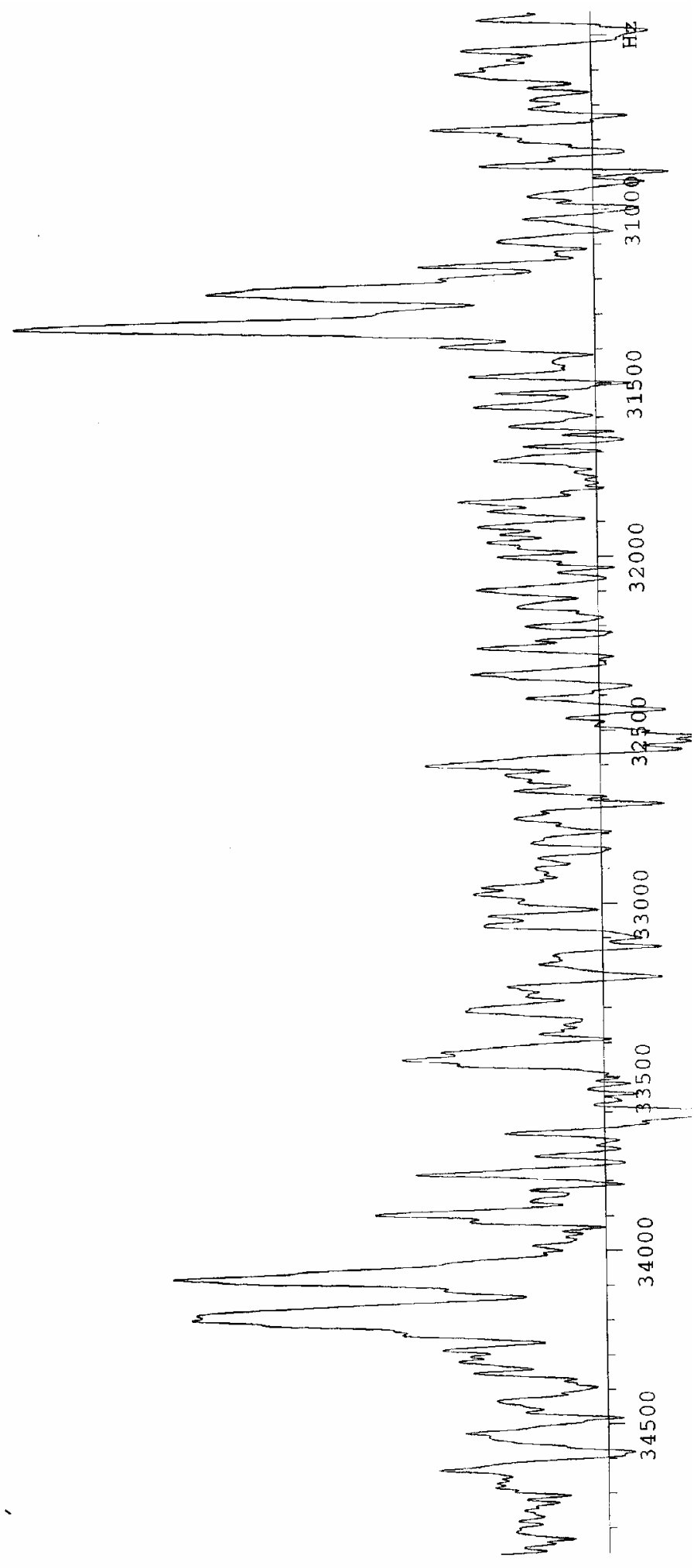


Figure S5. ${ }^{13} \mathrm{C}$-NMR spectrum of $\mathbf{4}$ - Expanded region showing $\alpha$-carbon resonances.

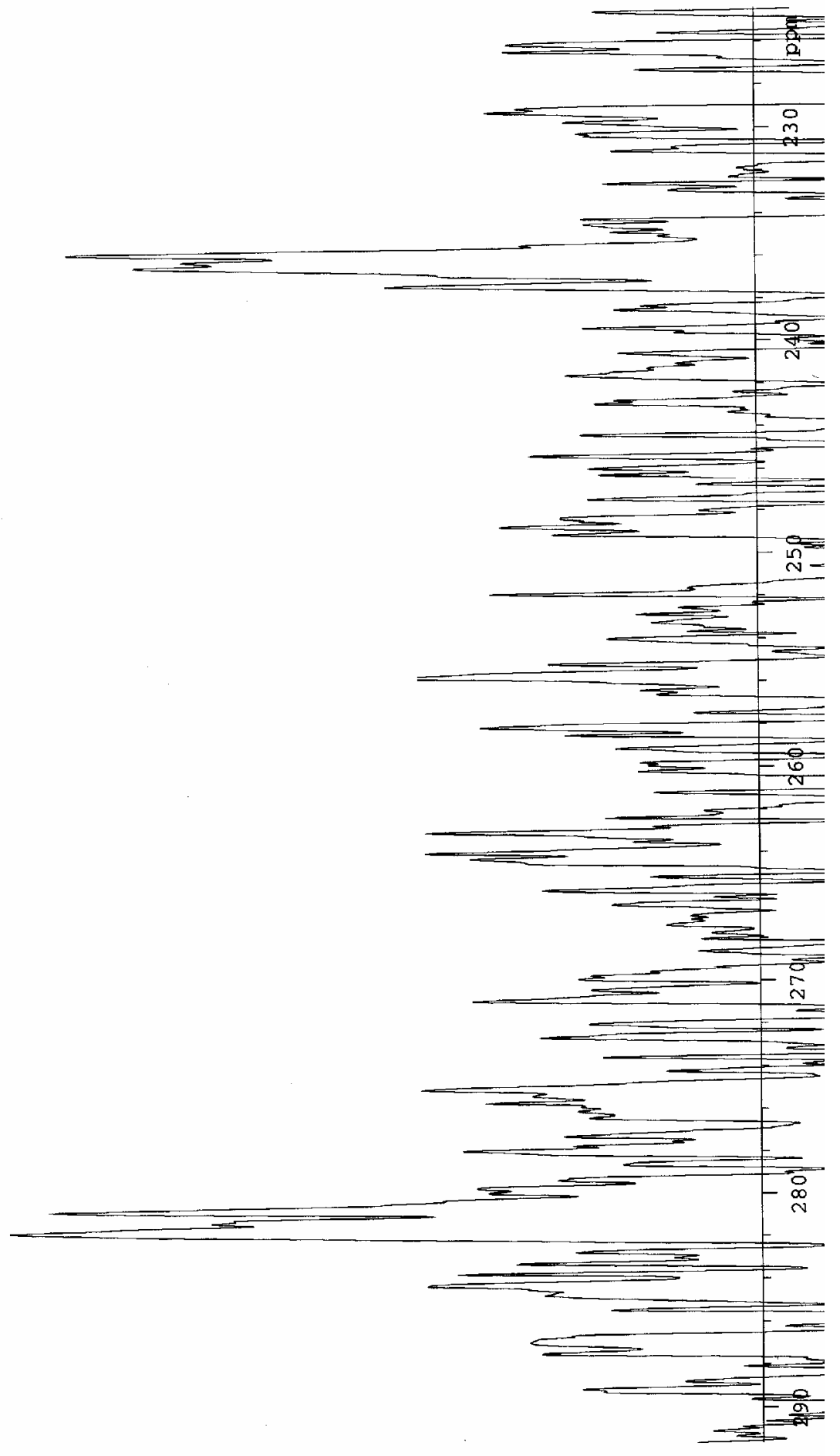


Figure S6. ${ }^{13} \mathrm{C}$-NMR spectrum of 5 - Expanded region showing $\alpha$-carbon resonances.

\section{Computational Details}

All calculations were carried out using Density Functional Theory as implemented in the Jaguar 6.0 suite $^{5}$ of ab initio quantum chemistry programs. Geometry optimizations were performed with the $\mathrm{B} 3 \mathrm{LYP}^{6-9}$ functional and the $6-31 \mathrm{G}^{* *}$ basis set with no symmetry restrictions. Niobium was represented using the Los Alamos LACVP basis $^{10-12}$. The energies of the optimized structures were reevaluated by additional singlepoint calculations on each optimized geometry using Dunning's correlation-consistent triple- $\xi$ basis set ${ }^{13}$ cc-pVTZ(-f) that includes a double set of polarization functions. For all transition metals, we used a modified version of LACVP, designated as LACV3P, in which the exponents were decontracted to match the effective core potential with the triple- $\xi$ quality basis.

NMR shielding constants are calculated by the method implemented in Jaguar 6.0, with B3LYP and the same basis sets as for single point calculation mentioned above. The ${ }^{13} \mathrm{C}$ and $1 \mathrm{H}$ chemical shifts are calculated as follows:

$$
\delta(\mathrm{ppm})=\sigma\left(\mathrm{Si}\left(\mathrm{CH}_{3}\right)_{4}\right)-\sigma_{s}
$$

$\sigma\left(\mathrm{Si}\left(\mathrm{CH}_{3}\right)_{4}\right)$ is the NMR shielding tenser in $\mathrm{Si}\left(\mathrm{CH}_{3}\right)_{4}$, and $\sigma_{\mathrm{s}}$ is the shielding tensor of the atom under investigation.

The models used in this study consist of $\sim 100$ atoms, which represent the nontruncated substrates that were also used in the experimental work. Although a smaller model may also able to reproduce the most important features of the studied reaction qualitatively, we chose to employ the large scale model to faithfully construct realistic model chemistry. These calculations challenge the current state of computational capabilities, whereas the numerical efficiency of the Jaguar program allows us to accomplish this task in a bearable time frame. 
S5. Calculated isomers and their relative electronic energies (in $\mathrm{kcal} / \mathrm{mol}, \mathbf{R}=\mathbf{T M S}$ )<smiles>[R]C=[W]</smiles>

71

$+3.69$<smiles></smiles>

73

0.00

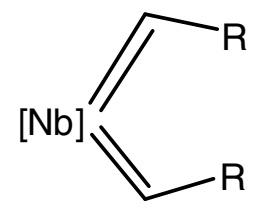

72

$+3.62$

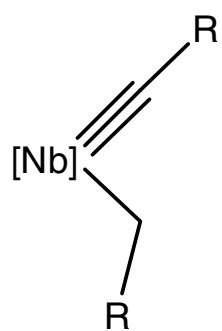

77

$+13.67$

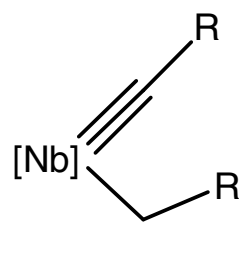

64

\begin{tabular}{|l|l|l|l|}
\hline & $\begin{array}{l}\text { Carbon Shielding } \\
\text { tensor }\end{array}$ & $\begin{array}{l}\alpha \text {-Carbon Chemical } \\
\text { Shifts }(\mathrm{ppm})\end{array}$ & $\begin{array}{l}\text { Gap of the two } \\
\text { absorptions }(\mathrm{ppm})\end{array}$ \\
\hline $\mathbf{7 1}$ & $-80.9,-92.0$ & $264.5,275.6$ & 11.5 \\
\hline $\mathbf{7 3}$ & $\mathbf{- 7 6 . 9 , - 1 0 1 . 3}$ & $\mathbf{2 6 0 . 5 , 2 8 4 . 9}$ & $\mathbf{2 4 . 4}$ \\
\hline $\mathbf{7 2}$ & $-91.9,-93.0$ & $275.5,276.6$ & 1.1 \\
\hline $\mathbf{7 7}$ & $129.2,-186.4$ & $54.4,370.0$ & 315.6 \\
\hline $\mathbf{6 4}$ & $85.7,-180.2$ & $97.9,363.8$ & 265.9 \\
\hline Complex 4 & & $\mathbf{2 4 9 . 7 , 2 7 2 . 2}$ & $\mathbf{2 2 . 5}$ \\
\hline
\end{tabular}

\begin{tabular}{|l|l|l|l|}
\hline & Proton Shielding tensor & $\begin{array}{l}\alpha \text {-Proton Chemical } \\
\text { Shifts (ppm) }\end{array}$ & $\begin{array}{l}\text { Gap of the } \alpha-\mathrm{H} \\
\text { absorptions }(\mathrm{ppm})\end{array}$ \\
\hline $\mathbf{7 1}$ & $17.2,16.2$ & $14.4,15.4$ & 1.0 \\
\hline $\mathbf{7 3}$ & $\mathbf{2 0 . 4 , 1 7 . 7}$ & $\mathbf{1 1 . 2 , 1 3 . 9}$ & $\mathbf{2 . 7}$ \\
\hline $\mathbf{7 2}$ & $19.2,19.1$ & $12.4,12.5$ & 0.1 \\
\hline $\mathbf{7 7}$ & $32.6,28.1$ & $-1.0,3.5$ & 4.5 \\
\hline $\mathbf{6 4}$ & $35.6,27.5$ & $-4.0,4.1$ & 8.1 \\
\hline Complex 4 & & $\mathbf{7 . 5 2 , 1 2 . 1 7}$ & $\mathbf{4 . 6 5}$ \\
\hline
\end{tabular}

The experimental ${ }^{13} \mathrm{C}-\mathrm{NMR}$ shifts agree best with those calculated for structure $\mathbf{7 3}$ when considering both calculated shifts and difference in shifts for the $\alpha$-Carbons. 
S6. Calculated isomers and their relative electronic energies (in $\mathbf{k c a l} / \mathbf{m o l}, \mathbf{R}={ }^{\mathrm{t}} \mathrm{Bu}$ )<smiles></smiles>

81

$+5.93$<smiles></smiles>

80

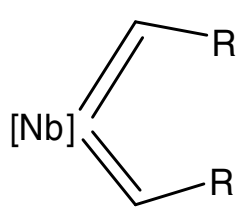

82

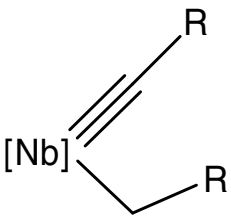

87

\begin{tabular}{|l|l|l|l|}
\hline & $\begin{array}{l}\text { Carbon Shielding } \\
\text { tensor }\end{array}$ & $\begin{array}{l}\alpha \text {-Carbon Chemical } \\
\text { Shift }(\mathrm{ppm})\end{array}$ & $\begin{array}{l}\text { Gap of the } \alpha-C \\
\text { absorptions }(\mathrm{ppm})\end{array}$ \\
\hline $\mathbf{8 1}$ & $-87.7,-112.8$ & $271.3,296.4$ & 25.1 \\
\hline $\mathbf{8 0}$ & $\mathbf{- 6 4 . 9 , - 1 1 8 . 4}$ & $\mathbf{2 4 8 . 5 , 3 0 2 . 0}$ & $\mathbf{5 3 . 5}$ \\
\hline $\mathbf{8 2}$ & $-103.0,-103.4$ & $286.6,287.0$ & 0.4 \\
\hline $\mathbf{8 7}$ & $71.7,-155.6$ & $111.9,339.2$ & 227.3 \\
\hline Complex 5 & & $\mathbf{2 3 7 . 1 , 2 8 2 . 5}$ & $\mathbf{4 5 . 4}$ \\
\hline
\end{tabular}

\begin{tabular}{|l|l|l|l|}
\hline & Proton Shielding tensor & $\begin{array}{l}\alpha \text {-Proton Chemical } \\
\text { Shift }(\mathrm{ppm})\end{array}$ & $\begin{array}{l}\text { Gap of the } \alpha-\mathrm{H} \\
\text { absorptions }(\mathrm{ppm})\end{array}$ \\
\hline $\mathbf{8 1}$ & $19.4,17.8$ & 12.213 .8 & 1.6 \\
\hline $\mathbf{8 0}$ & $\mathbf{2 5 . 6 1 8 . 8}$ & $\mathbf{6 . 0 , 1 2 . 8}$ & $\mathbf{6 . 8}$ \\
\hline $\mathbf{8 2}$ & $21.6,21.5$ & $10.0,10.1$ & 0.1 \\
\hline $\mathbf{8 7}$ & $30.8,30.4$ & $0.8,1.2$ & 0.4 \\
\hline Complex 5 & & $\mathbf{2 . 2 , 1 0 . 9 7}$ & $\mathbf{8 . 7 7}$ \\
\hline
\end{tabular}

The experimental ${ }^{13} \mathrm{C}$-NMR shifts agree best with those calculated for structure $\mathbf{8 0}$ when considering both calculated shifts and he $\alpha$-Carbons shift difference. 


\section{S7. Optimized Structures}

\begin{tabular}{|c|c|c|c|}
\hline 71 & & & \\
\hline $\mathrm{Nb}$ & -0.669227160 & 0.161241672 & -0.47528551 \\
\hline $\mathrm{P}$ & -0.984295342 & -2.252852468 & 0.589076769 \\
\hline $\mathrm{C}$ & 0.783506398 & -2.705909606 & 0.685186848 \\
\hline $\mathrm{Si}$ & -0.000813072 & -0.838915613 & -3.964237481 \\
\hline $\mathrm{C}$ & 1.273528700 & -4.018578123 & 0.702651811 \\
\hline $\mathrm{H}$ & 0.570822328 & -4.848651783 & 0.675599890 \\
\hline $\mathrm{C}$ & 2.640620909 & -4.301915145 & 0.741871042 \\
\hline $\mathrm{C}$ & 3.160868406 & -5.720867208 & 0.741460140 \\
\hline $\mathrm{H}$ & 2.343371677 & -6.446042126 & 0.801506199 \\
\hline $\mathrm{H}$ & 3.831256988 & -5.905520214 & 1.589717714 \\
\hline $\mathrm{H}$ & 3.730920494 & -5.941765769 & -0.169934734 \\
\hline $\mathrm{C}$ & 3.516644535 & -3.206055499 & 0.758859186 \\
\hline $\mathrm{H}$ & 4.590879140 & -3.383021369 & 0.768558829 \\
\hline $\mathrm{C}$ & 3.055336790 & -1.895750184 & 0.749852805 \\
\hline $\mathrm{H}$ & 3.770471002 & -1.079608135 & 0.755320061 \\
\hline $\mathrm{C}$ & 1.673735794 & -1.600105948 & 0.729108567 \\
\hline $\mathrm{N}$ & 1.172524512 & -0.282684001 & 0.692092274 \\
\hline $\mathrm{C}$ & 1.852755017 & 0.720897472 & 1.406200391 \\
\hline $\mathrm{C}$ & 2.554493640 & 0.464425918 & 2.607762796 \\
\hline $\mathrm{H}$ & 2.596721008 & -0.550020804 & 2.989426389 \\
\hline $\mathrm{C}$ & 3.182230434 & 1.480884264 & 3.314804115 \\
\hline $\mathrm{H}$ & 3.699994612 & 1.232656913 & 4.239889351 \\
\hline $\mathrm{C}$ & 3.152930161 & 2.814880220 & 2.880691293 \\
\hline $\mathrm{C}$ & 3.841825522 & 3.908641989 & 3.663763508 \\
\hline $\mathrm{H}$ & 3.649288128 & 4.894131903 & 3.228384402 \\
\hline $\mathrm{H}$ & 4.929668426 & 3.765547312 & 3.688115718 \\
\hline $\mathrm{H}$ & 3.500431497 & 3.934626001 & 4.705981311 \\
\hline $\mathrm{C}$ & 2.450244023 & 3.079708003 & 1.703718738 \\
\hline $\mathrm{H}$ & 2.397018150 & 4.107751767 & 1.352097764 \\
\hline $\mathrm{C}$ & 1.802765063 & 2.072932125 & 0.972970433 \\
\hline $\mathrm{P}$ & 0.841424253 & 2.368777595 & -0.553470958 \\
\hline $\mathrm{C}$ & -1.595253163 & -2.351079336 & 2.380754944 \\
\hline $\mathrm{H}$ & -2.613586314 & -1.947088146 & 2.332117503 \\
\hline $\mathrm{C}$ & -0.757160044 & -1.440213796 & 3.291307284 \\
\hline $\mathrm{H}$ & -1.202416532 & -1.407794099 & 4.293042697 \\
\hline $\mathrm{H}$ & -0.692422255 & -0.417517637 & 2.914531382 \\
\hline $\mathrm{H}$ & 0.263343806 & -1.823006503 & 3.393827490 \\
\hline $\mathrm{C}$ & -1.645869936 & -3.776461081 & 2.952063305 \\
\hline $\mathrm{H}$ & -0.657346018 & -4.248336927 & 2.926801261 \\
\hline $\mathrm{H}$ & -2.348916882 & -4.424154903 & 2.420353329 \\
\hline $\mathrm{H}$ & -1.965326694 & -3.741496068 & 4.000796859 \\
\hline $\mathrm{C}$ & -1.876634642 & -3.676028693 & -0.254347562 \\
\hline $\mathrm{H}$ & -1.673737200 & -4.574364139 & 0.341449859 \\
\hline $\mathrm{C}$ & -3.392811034 & -3.417055499 & -0.249083908 \\
\hline $\mathrm{H}$ & -3.633108768 & -2.496572743 & -0.791053046 \\
\hline $\mathrm{H}$ & -3.801969808 & -3.327926381 & 0.761992480 \\
\hline $\mathrm{H}$ & -3.912215948 & -4.245727238 & -0.744169470 \\
\hline $\mathrm{C}$ & -1.366698064 & -3.911450758 & -1.679948929 \\
\hline $\mathrm{H}$ & -1.922756979 & -4.736982582 & -2.140537709 \\
\hline $\mathrm{H}$ & -0.304355122 & -4.168629478 & -1.699928884 \\
\hline $\mathrm{H}$ & -1.511337335 & -3.016849218 & -2.290134735 \\
\hline $\mathrm{C}$ & 2.149790559 & 2.538448630 & -1.912456284 \\
\hline $\mathrm{H}$ & 1.561486189 & 2.557098692 & -2.837632061 \\
\hline $\mathrm{C}$ & 3.051019292 & 1.294623233 & -1.932382040 \\
\hline $\mathrm{H}$ & 3.704165546 & 1.319110368 & -2.812829690 \\
\hline $\mathrm{H}$ & 2.474162865 & 0.368441783 & -1.961504401 \\
\hline $\mathrm{H}$ & 3.689724821 & 1.263732063 & -1.043591092 \\
\hline $\mathrm{C}$ & 3.001048650 & 3.814336842 & 1831256345 \\
\hline
\end{tabular}

H $\quad 3.540026108 \quad 3.873024001 \quad-0.879343236$

H $2.411902083 \quad 4.728336585 \quad-1.950157430$

H $3.751931903 \quad 3.805458095 \quad-2.630980326$

C $0.139162938 \quad 4.105041806 \quad-0.401521952$

H $\quad 0.996682996 \quad 4.784164676 \quad-0.320941343$

C $\quad-0.727452956 \quad 4.267665721 \quad 0.852243222$

H $\quad-1.113201421 \quad 5.2929207390 .905733634$

H $\quad-0.165627329 \quad 4.070130258 \quad 1.769387173$

H $\quad-1.577833474 \quad 3.582963337 \quad 0.819239330$

C $\quad-0.651092941 \quad 4.461103635-1.671562165$

H $\quad-1.5001814893 .782149794-1.802409552$

$\mathrm{H} \quad-0.036444899 \quad 4.412959067 \quad-2.576199620$

H $\quad-1.044102760 \quad 5.481373694 \quad-1.592745081$

C $\quad-2.393784230 \quad 0.895498893 \quad 0.127214247$

$\begin{array}{lllll}\text { Si } & -3.647676660 & 1.439486929 & 1.399882633\end{array}$

C $\quad-2.893425580 \quad 1.805528826 \quad 3.102878131$

$\begin{array}{llll}\mathrm{H} & -3.664964800 & 2.175791819 & 3.788855691\end{array}$

$\mathrm{H} \quad-2.107247193 \quad 2.565683266 \quad 3.047506110$

H $\quad-2.455595278 \quad 0.910121439 \quad 3.555594138$

C $\quad-4.572470559 \quad 2.994827665 \quad 0.804241174$

H $\quad-5.058279206 \quad 2.816047269-0.162451926$

H $\quad-3.907873305 \quad 3.856845156 \quad 0.683637672$

$\begin{array}{llll}\mathrm{H} & -5.355175236 & 3.278338500 & 1.518946947\end{array}$

$\begin{array}{llll}\text { C } & -4.974232578 & 0.089871959 & 1.624385392\end{array}$

H $\quad-4.557869484-0.828830502 \quad 2.052097889$

$\begin{array}{llll}\mathrm{H} & -5.435500612 & -0.173810048 & 0.665597083\end{array}$

$\begin{array}{llll}\mathrm{H} & -5.772804137 & 0.433394170 & 2.293716967\end{array}$

C $\quad-0.828946840 \quad-0.204004285-2.408852602$

C $\quad 0.597304493 \quad 0.611533242 \quad-5.047284623$

H $\quad 1.425869329 \quad 1.154186058$-4.580464486

H $\quad-0.210221068 \quad 1.330057415 \quad-5.230301959$

H $\quad 0.947153885 \quad 0.251492583 \quad-6.023197463$

C $-1.264964006-1.784980775-5.032128138$

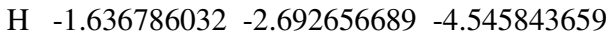

H $\quad-0.813323308-2.083785159-5.986444531$

H $\quad-2.132918858-1.155311137-5.262430357$

C $\quad 1.481767860-1.981604490 \quad-3.642207990$

H $\quad 1.220771114-2.810875583-2.977510988$

H $\quad 2.324127555-1.450640257 \quad-3.187231882$

H $\quad 1.839448476-2.411650982-4.586130523$

$\begin{array}{lllll}\mathrm{H} & -2.890203341 & 0.860845238 & -0.863707743\end{array}$

H $\quad-1.833700328 \quad 0.169431335 \quad-2.672656435$

$==$ 
C $\quad 2.434711382 \quad 0.624902329 \quad 2.941743667$

H $\quad 2.428985290 \quad-0.338610342 \quad 3.440351822$

C $\quad 2.963990405 \quad 1.726641882 \quad 3.599778965$

H $\quad 3.359559199 \quad 1.595874983 \quad 4.605569839$

C $\quad 2.986872858 \quad 3.001424208 \quad 3.013359374$

C $\quad 3.568044401 \quad 4.189746752 \quad 3.744192404$

$\mathrm{H} \quad 3.424404373 \quad 5.115767788 \quad 3.178951784$

H $4.645361286 \quad 4.072290349 \quad 3.916734192$

H $\quad 3.100743745 \quad 4.326179041 \quad 4.727182561$

C $\quad 2.432254098 \quad 3.118541132 \quad 1.738052945$

$\begin{array}{llll}\mathrm{H} & 2.413524248 & 4.099734049 & 1.268816713\end{array}$

C $\quad 1.882814334 \quad 2.023738942 \quad 1.055738314$

$\begin{array}{lllll}\mathrm{P} & 1.051082303 & 2.138339386 & -0.566131801\end{array}$

C $-1.677671671 \quad-2.659295702 \quad 2.213693828$

H $\quad-2.695124053-2.374682443 \quad 1.918190593$

C $-1.171027234 \quad-1.636726878 \quad 3.243672357$

H $-1.817558518-1.647447499 \quad 4.129211880$

H $-1.161333182-0.620416160 \quad 2.842325588$

$\begin{array}{lllll}\mathrm{H} & -0.154326418 & -1.879757875 & 3.569821121\end{array}$

$\begin{array}{llll}\text { C } & -1.703932321 & -4.072862597 & 2.812887419\end{array}$

H $\quad-0.693331309 \quad-4.419942836 \quad 3.054224927$

H $\quad-2.168521242-4.806515735 \quad 2.147087075$

H $\quad-2.281093946-4.068414488 \quad 3.745564837$

C $-1.216330597 \quad-3.981524181 \quad-0.415216333$

H $\quad-1.102071518 \quad-4.864385786 \quad 0.226091012$

$\begin{array}{llll}\text { C } & -2.697636077 & -3.849579568 & -0.803927417\end{array}$

H $\quad-2.861304491 \quad-2.954204109-1.412093763$

H $\quad-3.356840868 \quad-3.7899538850 .068165380$

H $\quad-3.006532294 \quad-4.720522727-1.393641596$

C $-0.329831886-4.163185372-1.654787248$

H $\quad-0.673646988-5.031532261-2.229318310$

H $\quad 0.717636861 \quad-4.328679371-1.388860231$

H $\quad-0.381754761 \quad-3.282890898 \quad-2.300803195$

C $\quad 2.445675529 \quad 2.176313454 \quad-1.849622387$

H $\quad 1.917877845 \quad 2.109939482-2.809026543$

C $3.342616444 \quad 0.936738754-1.707827528$

H $\quad 4.080059683 \quad 0.919966017 \quad-2.518884521$

H $\quad 2.773192806 \quad 0.005832227-1.749806718$

$\begin{array}{lllll}\mathrm{H} & 3.889410685 & 0.955284819 & -0.759608218\end{array}$

C $3.2954675163 .456511025-1.825576568$

H $3.772389822 \quad 3.598096021 \quad-0.849372055$

H $\quad 2.717325919 \quad 4.355462866-2.058206946$

H $\quad 4.095380052 \quad 3.382754333 \quad-2.572398940$

C $\quad 0.315183425 \quad 3.865763669-0.641936375$

H $\quad 1.148350955 \quad 4.570156036-0.529082007$

C $\quad-0.681994530 \quad 4.105402219 \quad 0.498445223$

$\mathrm{H} \quad-1.088307205 \quad 5.121491987 \quad 0.428258263$

$\begin{array}{lllll}\mathrm{H} & -0.217802681 & 3.994728630 & 1.482085464\end{array}$

$\mathrm{H} \quad-1.513284156 \quad 3.399744634 \quad 0.433184073$

C $\quad-0.343506218 \quad 4.103328872-2.009918729$

H $-1.162997742 \quad 3.396259614-2.173876568$

H $\quad 0.363253159 \quad 4.003134126-2.839767826$

H $\quad-0.759492599 \quad 5.116651549-2.051540558$

C $\quad-2.2165283790 .567207826-0.099565102$

$\begin{array}{llll}\mathrm{Si} & -3.692634755 & 1.213819312 & 0.839229350\end{array}$

C $\quad-3.225234382 \quad 1.798466582 \quad 2.584307644$

H $\quad-4.102646036 \quad 2.201689900 \quad 3.104556997$

$\mathrm{H} \quad-2.461752841 \quad 2.583248756 \quad 2.565013832$

H $\quad-2.834756170 \quad 0.974310664 \quad 3.191122046$

C $-4.511906062 \quad 2.653940138-0.097804919$

H $\quad-4.8244357362 .340660801-1.100921426$

H $\quad-3.837178703 \quad 3.508494127-0.214418155$
H $\quad-5.405716002 \quad 3.006976781 \quad 0.431240220$

C $\quad-5.006668650-0.154374344 \quad 1.013450733$

H $\quad-4.629701759-0.999106381 \quad 1.601327073$

H $\quad-5.307841846 \quad-0.5428596390 .033660423$

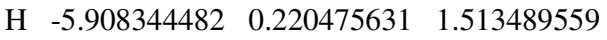

C $-0.231953218-0.564029940-2.339393287$

C $\quad-0.223923620 \quad 0.934760623 \quad-5.033904078$

$\mathrm{H} \quad 0.847687870 \quad 0.735087542 \quad-5.150709087$

H $\quad-0.330319448 \quad 1.916188641 \quad-4.558651251$

$\begin{array}{lllll}\mathrm{H} & -0.658945167 & 1.002738149 & -6.038874886\end{array}$

C $\quad-2.915674065 \quad 0.013778863 \quad-3.865471908$

$\mathrm{H} \quad-3.071499358 \quad 0.982585914 \quad-3.378347070$

H $\quad-3.472010951-0.738357793 \quad-3.294079482$

$\begin{array}{lllll}\mathrm{H} & -3.368747232 & 0.070195578 & -4.862793678\end{array}$

C $-0.923429288-2.043878843 \quad-4.990332956$

H $\quad 0.124374893 \quad-2.347392383 \quad-5.100578245$

H $\quad-1.341434535-1.929685285 \quad-5.998290552$

H $\quad-1.455283236-2.869097974-4.504216534$

H $\quad-2.467997155 \quad 0.374930212 \quad-1.171009663$

H $\quad 0.794039395 \quad-0.970640216-2.433141361$

\section{2}

$\begin{array}{lllll}\mathrm{Nb} & -0.394094389 & 0.067753914 & -0.270779746\end{array}$

P $\quad-0.772562616 \quad-2.308242250 \quad 0.855107192$

C $\quad 0.968654010 \quad-2.776781702 \quad 1.144033225$

$\begin{array}{llll}\mathrm{Si} & -1.324690907 & -0.168814828 & -3.832654564\end{array}$

$\begin{array}{llll}\text { C } & 1.416340120 & -4.095927018 & 1.315091273\end{array}$

H $\quad 0.689368748 \quad-4.904498762 \quad 1.335868250$

C $\quad 2.768682338-4.415534073 \quad 1.441234829$

C $3.241691348 \quad-5.839693944 \quad 1.618081536$

H $\quad 2.399651375-6.536206551 \quad 1.675346700$

H $3.832310553-5.957692387 \quad 2.534911335$

H $\quad 3.879472751 \quad-6.159984807 \quad 0.784559019$

C $\quad 3.680405315 \quad-3.349933903 \quad 1.368904094$

H $\quad 4.747554162-3.556518164 \quad 1.430137790$

C $3.262870260 \quad-2.036388071 \quad 1.208076585$

H $\quad 4.004489612-1.246577071 \quad 1.150761347$

C $1.891041939-1.699232500 \quad 1.116943034$

N $\quad 1.441997209 \quad-0.375136201 \quad 0.948162429$

$\begin{array}{llll}\text { C } & 2.175386411 & 0.655436102 & 1.565420468\end{array}$

$\begin{array}{llll}\text { C } & 2.848857643 & 0.482185162 & 2.799936700\end{array}$

H $\quad 2.809873161 \quad-0.484824187 \quad 3.289891655$

C $\quad 3.549726562 \quad 1.518235190 \quad 3.400618117$

H $\quad 4.043346479 \quad 1.335526787 \quad 4.353380126$

$\begin{array}{llll}\text { C } & 3.622523342 & 2.795530444 & 2.821247889\end{array}$

C $\quad 4.393948642 \quad 3.913898591 \quad 3.483361175$

$\mathrm{H} \quad 4.258217868 \quad 4.8614377992 .952849906$

$\mathrm{H} \quad 5.470555968 \quad 3.702846587 \quad 3.510447697$

$\mathrm{H} \quad 4.072855876 \quad 4.066349303 \quad 4.521020902$

C $\quad 2.936361043 \quad 2.984363261 \quad 1.621318026$

H $\quad 2.951771529 \quad 3.972401713 \quad 1.167081319$

$\begin{array}{llll}\text { C } & 2.215354126 & 1.955113278 & 0.996479644\end{array}$

P $\quad 1.214122427 \quad 2.174504156 \quad-0.516046620$

C $\quad-1.605346963 \quad-2.345843614 \quad 2.553364943$

H $\quad-2.620610919-1.980292865 \quad 2.353965088$

C $-0.915318605 \quad-1.354107906 \quad 3.503877710$

H $\quad-1.458457146-1.312536283 \quad 4.455168778$

H $\quad-0.881449121 \quad-0.343227170 \quad 3.090932465$

H $\quad 0.111803911-1.666865404 \quad 3.718622167$

C $-1.685509375-3.740318721 \quad 3.192604692$

H $\quad-0.686583206-4.162151976 \quad 3.347692918$ 
H $\quad-2.267846992-4.448324164 \quad 2.595721601$

$\mathrm{H} \quad-2.167079474 \quad-3.668876724 \quad 4.175412165$

C $-1.522696662-3.761102576-0.074262922$

H $\quad-1.390386323 \quad-4.641077636 \quad 0.567336718$

$\begin{array}{llll}\text { C } & -3.026477957 & -3.541550950 & -0.298588668\end{array}$

H $\quad-3.200560483-2.659008551 \quad-0.920901778$

H $\quad-3.576996724 \quad-3.410537348 \quad 0.638761274$

H $\quad-3.455679057-4.407937743-0.815023151$

C $-0.797800962-4.015799409-1.403380440$

H $-1.252062758-4.874868515-1.911388535$

H $\quad 0.263845066-4.233514790-1.256843732$

H $\quad-0.881756167-3.146071215 \quad-2.061095864$

C $2.446211050 \quad 2.187268485-1.952550280$

$\begin{array}{lllll}\mathrm{H} & 1.806243309 & 2.223683870 & -2.843298257\end{array}$

C $\quad 3.238823334 \quad 0.870910532-1.984323707$

$\begin{array}{lllll}\mathrm{H} & 3.884079556 & 0.847570680 & -2.870184603\end{array}$

H $\quad 2.583179450 \quad-0.002074462-2.021785331$

H $\quad 3.879426091 \quad 0.777907674-1.101126035$

C $3.398884324 \quad 3.392288895 \quad-1.964669237$

H $\quad 4.010757506 \quad 3.422487666-1.056608822$

$\mathrm{H} \quad 2.874694350 \quad 4.348000803 \quad-2.056620224$

H $\quad 4.083199772 \quad 3.312876264-2.818115710$

C $\quad 0.580466798 \quad 3.944894622-0.433348078$

H $\quad 1.467773825 \quad 4.588498519-0.389191807$

C $\quad-0.257684068 \quad 4.1918872820 .828773754$

$\mathrm{H} \quad-0.592290284 \quad 5.236048775 \quad 0.849483937$

H $\quad 0.311743672 \quad 4.002387798 \quad 1.743022236$

H $\quad-1.142658395 \quad 3.549714367 \quad 0.836354007$

C $\quad-0.212415630 \quad 4.294735569-1.702243644$

H $\quad-1.095247150 \quad 3.655723709-1.795686673$

H $\quad 0.383989146 \quad 4.186673620-2.614017199$

H $\quad-0.553779771 \quad 5.335297967 \quad-1.653166503$

C $\quad-1.996449498 \quad 0.940343593 \quad 0.467289025$

$\begin{array}{llll}\mathrm{Si} & -3.809042660 & 1.346096420 & 0.251748016\end{array}$

C $\quad-4.667598176 \quad 1.212315203 \quad 1.946851676$

$\mathrm{H} \quad-5.738809984 \quad 1.436105357 \quad 1.871237478$

$\mathrm{H} \quad-4.234360574 \quad 1.910824783 \quad 2.672391032$

$\begin{array}{lllll}\mathrm{H} & -4.570545936 & 0.202736755 & 2.363468349\end{array}$

C $-4.080106593 \quad 3.119656158-0.384481900$

H $-3.623406520 \quad 3.267894011-1.369151346$

H $\quad-3.645765969 \quad 3.861151543 \quad 0.295652754$

$\begin{array}{llll}\text { H } & -5.150495837 & 3.341887342 & -0.477430829\end{array}$

C $\quad-4.696773215 \quad 0.152123531-0.926705551$

$\mathrm{H} \quad-4.723015480 \quad-0.859711284-0.507838515$

H $\quad-4.205316463 \quad 0.092003469-1.902362108$

$\begin{array}{lllll}\mathrm{H} & -5.733642547 & 0.468679122 & -1.094458026\end{array}$

$\begin{array}{llll}\text { C } & -0.496092467 & -0.408378052 & -2.174449468\end{array}$

$\begin{array}{lllll}\text { C } & -2.271574026 & 1.470446953 & -3.951778995\end{array}$

$\mathrm{H} \quad-1.5891381132 .321779525 \quad-3.854816029$

$\mathrm{H} \quad-3.031381825 \quad 1.565922972-3.170364836$

$\begin{array}{llll}\mathrm{H} & -2.777552171 & 1.558366853 & -4.920961013\end{array}$

C $-2.524525412-1.584644294-4.249187956$

H $\quad-2.011505016 \quad-2.552982043 \quad-4.266057727$

H $\quad-2.980625560-1.433351630 \quad-5.235419787$

Н $\quad-3.334448334-1.654262549-3.514691097$

C $\quad 0.019435097 \quad-0.144501338-5.182191981$

H $\quad 0.572961567 \quad-1.090333600-5.214169112$

H $\quad 0.746778490 \quad 0.656356400 \quad-5.004505259$

H $\quad-0.415039667 \quad 0.018831715-6.176268664$

H $\quad-1.673687617 \quad 1.204974985 \quad 1.496075328$

H $\quad 0.446206662 \quad-0.982359346-2.303884165$
77

$\begin{array}{lllll}\mathrm{Nb} & -0.585452209 & 0.113285423 & -0.397493336\end{array}$

$\begin{array}{llll}\text { P } & -0.868181260 & -2.344331444 & 0.690492344\end{array}$

$\begin{array}{lrrrr}\text { C } & 0.917587144 & -2.699748855 & 0.863798467\end{array}$

$\begin{array}{lllll}\mathrm{Si} & -0.116379651 & -0.759912764 & -3.916517767\end{array}$

$\begin{array}{lllll}\text { C } & 1.444591501 & -3.999560258 & 0.901351002\end{array}$

$\begin{array}{lllll}\text { H } & 0.772192258 & -4.850004970 & 0.816879508\end{array}$

$\begin{array}{llll}\text { C } & 2.811371786 & -4.244815161 & 1.039152955\end{array}$

$\begin{array}{llll}\text { C } & 3.369672147 & -5.648796716 & 1.074232737\end{array}$

$\begin{array}{llll}\text { H } & 2.573682288 & -6.397360958 & 1.015426838\end{array}$

H $\quad 3.933969623 \quad-5.836538026 \quad 1.995993359$

$\begin{array}{lllll}\mathrm{H} & 4.055910608 & -5.830313381 & 0.237674351\end{array}$

$\begin{array}{llll}\text { C } & 3.654230321 & -3.125876502 & 1.131969557\end{array}$

$\begin{array}{llll}\mathrm{H} & 4.728848281 & -3.274502360 & 1.221331701\end{array}$

$\begin{array}{llll}\text { C } & 3.157895980 & -1.830730374 & 1.097099640\end{array}$

H $3.845911734-0.993934047 \quad 1.159073275$

$\begin{array}{llll}\text { C } & 1.773393725 & -1.570715269 & 0.965827413\end{array}$

$\begin{array}{llll}\mathrm{N} & 1.245963989 & -0.275252548 & 0.887929947\end{array}$

$\begin{array}{llll}\text { C } & 1.850567773 & 0.748966065 & 1.626450878\end{array}$

$\begin{array}{llll}\text { C } & 2.443741762 & 0.547013073 & 2.895131895\end{array}$

$\begin{array}{llll}\text { H } & 2.454575693 & -0.451905760 & 3.318725071\end{array}$

$\begin{array}{llll}\text { C } & 2.997528341 & 1.598884614 & 3.611919562\end{array}$

$\begin{array}{llll}\mathrm{H} & 3.431645527 & 1.396052657 & 4.589396672\end{array}$

C $\quad 2.996873776 \quad 2.913914284 \quad 3.121381401$

C $3.603426276 \quad 4.046228290 \quad 3.917560943$

H $3.444632631 \quad 5.010668533 \quad 3.425503543$

H $\quad 4.685300245 \quad 3.916268449 \quad 4.046934051$

H $\quad 3.167936306 \quad 4.111502794 \quad 4.922103772$

C $\quad 2.406284458 \quad 3.124490519 \quad 1.873761630$

$\begin{array}{llll}\mathrm{H} & 2.384466357 & 4.136240068 & 1.475682364\end{array}$

$\begin{array}{llll}\text { C } & 1.839614572 & 2.080184859 & 1.129390119\end{array}$

$\begin{array}{lllll}\text { P } & 1.017783790 & 2.290418399 & -0.490823546\end{array}$

$\begin{array}{llll}\text { C } & -1.558585113 & -2.538312551 & 2.449340189\end{array}$

$\begin{array}{llll}\text { H } & -2.579838909 & -2.143434289 & 2.385533167\end{array}$

C $\quad-0.764018366-1.663726272 \quad 3.434418700$

H $\quad-1.263644995 \quad-1.654396715 \quad 4.410079640$

H $\quad-0.662520648 \quad-0.630022306 \quad 3.095539227$

$\begin{array}{lllll}\text { H } & 0.244678241 & -2.063681913 & 3.578897198\end{array}$

C $\quad-1.618296390 \quad-3.987172994 \quad 2.956456513$

$\begin{array}{llll}\mathrm{H} & -0.623903327 & -4.446788294 & 2.957037933\end{array}$

$\begin{array}{llll}\text { H } & -2.288567204 & -4.618176792 & 2.366013830\end{array}$

H $\quad-1.984675007-3.999744920 \quad 3.990127425$

C $-1.610546379-3.788762843-0.254862605$

$\begin{array}{lllll}\mathrm{H} & -1.407723118 & -4.686221116 & 0.342122081\end{array}$

$\begin{array}{llll}\text { C } & -3.134071877 & -3.615487132 & -0.370426250\end{array}$

H $-3.382316043-2.712781828-0.937942279$

$\begin{array}{lllll}\text { H } & -3.627897676 & -3.548619109 & 0.604099376\end{array}$

H $\quad-3.565799297-4.472856251 \quad-0.899556659$

$\begin{array}{lllll}\text { C } & -0.970950811 & -3.970982821 & -1.636190643\end{array}$

H $\quad-1.420133673-4.837528936-2.136463968$

H $\quad 0.107758322 \quad-4.134475675-1.573582055$

H $\quad-1.140658381 \quad-3.088994157-2.256342441$

$\begin{array}{llll}\text { C } & 2.413064496 & 2.326819881 & -1.764882094\end{array}$

$\begin{array}{lllll}\mathrm{H} & 1.884881341 & 2.409817568 & -2.721935504\end{array}$

C 3.1838055850 .997444328 -1.755928539

$\begin{array}{lllll}\mathrm{H} & 3.907460482 & 0.986338463 & -2.579904851\end{array}$

H $\quad 2.513532705 \quad 0.141867052-1.867195633$

$\begin{array}{lllll}\text { H } & 3.742252823 & 0.876240791 & -0.821662027\end{array}$

C $3.370776514 \quad 3.518001424-1.609812777$

H $\quad 3.853966662 \quad 3.512606760-0.626870715$

H $\quad 2.875745766 \quad 4.484651294 \quad-1.743356431$ 
$\begin{array}{crrr}\mathrm{H} & 4.163005562 & 3.452057951 & -2.365185050 \\ \mathrm{C} & 0.342631999 & 4.046837929 & -0.502737985 \\ \mathrm{H} & 1.197585218 & 4.709612065 & -0.320737237 \\ \mathrm{C} & -0.686345180 & 4.273955221 & 0.614044894 \\ \mathrm{H} & -1.010414632 & 5.321494240 & 0.611781219 \\ \mathrm{H} & -0.274817222 & 4.051243691 & 1.602146583 \\ \mathrm{H} & -1.574219961 & 3.653230023 & 0.467343276 \\ \mathrm{C} & -0.247284993 & 4.388322598 & -1.881670677 \\ \mathrm{H} & -1.078884705 & 3.722055906 & -2.134410568 \\ \mathrm{H} & 0.494093388 & 4.318698126 & -2.683212561 \\ \mathrm{H} & -0.632252015 & 5.414780265 & -1.876781688 \\ \mathrm{C} & -2.638599970 & 1.046658203 & -0.462046614 \\ \mathrm{Si} & -3.817250162 & 1.324017222 & 0.975729741 \\ \mathrm{C} & -2.949907172 & 1.664256801 & 2.636371769 \\ \mathrm{H} & -3.685224319 & 1.878842811 & 3.421609502 \\ \mathrm{H} & -2.275281444 & 2.525811438 & 2.579365645 \\ \mathrm{H} & -2.356908935 & 0.806552698 & 2.971837436 \\ \mathrm{C} & -4.963231097 & 2.810404209 & 0.648339327 \\ \mathrm{H} & -5.528169232 & 2.676686943 & -0.281418343 \\ \mathrm{H} & -4.394590513 & 3.743467787 & 0.553448238 \\ \mathrm{H} & -5.687245131 & 2.949396523 & 1.460811552 \\ \mathrm{C} & -4.929853117 & -0.201947821 & 1.201322868 \\ \mathrm{H} & -4.345998889 & -1.112769108 & 1.371731374 \\ \mathrm{H} & -5.545481616 & -0.374013838 & 0.310588724 \\ \mathrm{H} & -5.608565067 & -0.080891855 & 2.053821006 \\ \mathrm{C} & -0.290279863 & -0.441760413 & -2.093238937 \\ \mathrm{C} & 0.367890960 & 0.850856172 & -4.808427987 \\ \mathrm{H} & 1.377046543 & 1.174631035 & -4.532465804 \\ \mathrm{H} & -0.327363393 & 1.662751713 & -4.569208115 \\ \mathrm{H} & 0.355351813 & 0.708370169 & -5.896135608 \\ \mathrm{C} & -1.759490427 & -1.344807385 & -4.676062139 \\ \mathrm{H} & -2.548778502 & -0.605248979 & -4.501118115 \\ \mathrm{H} & -2.097695693 & -2.296004564 & -4.251185536 \\ \mathrm{H} & -1.663020985 & -1.483022521 & -5.760373643 \\ \mathrm{C} & 1.224271844 & -2.049567870 & -4.312085376 \\ \mathrm{H} & 2.199766513 & -1.730771485 & -3.928228499 \\ \mathrm{H} & 1.322571943 & -2.194667030 & -5.395472760 \\ \mathrm{H} & 0.997422687 & -3.022863577 & -3.864993220 \\ \mathrm{H} & -2.420376841 & 2.029950870 & -0.918192028 \\ \mathrm{H} & -3.193979692 & 0.511353304 & -1.246625332\end{array}$

$==$
64

\begin{tabular}{|c|c|c|c|}
\hline $\mathrm{Nb}$ & -0.585452209 & 0.113285423 & -0.397493336 \\
\hline $\mathrm{P}$ & -0.868181260 & -2.344331444 & 0.690492344 \\
\hline $\mathrm{C}$ & 0.917587144 & -2.699748855 & 0.863798467 \\
\hline $\mathrm{Si}$ & -0.116379651 & -0.759912764 & -3.916517767 \\
\hline $\mathrm{C}$ & 1.444591501 & -3.999560258 & 0.901351002 \\
\hline $\mathrm{H}$ & 0.772192258 & -4.850004970 & 0.816879508 \\
\hline $\mathrm{C}$ & 2.811371786 & -4.244815161 & 1.039152955 \\
\hline $\mathrm{C}$ & 3.369672147 & -5.648796716 & 1.074232737 \\
\hline $\mathrm{H}$ & 2.573682288 & -6.397360958 & 1.015426838 \\
\hline $\mathrm{H}$ & 3.933969623 & -5.836538026 & 1.995993359 \\
\hline $\mathrm{H}$ & 4.055910608 & -5.830313381 & 0.237674351 \\
\hline $\mathrm{C}$ & 3.654230321 & -3.125876502 & 1.131969557 \\
\hline $\mathrm{H}$ & 4.728848281 & -3.274502360 & 1.221331701 \\
\hline $\mathrm{C}$ & 3.157895980 & -1.830730374 & 1.097099640 \\
\hline $\mathrm{H}$ & 3.845911734 & -0.993934047 & 1.159073275 \\
\hline $\mathrm{C}$ & 1.773393725 & -1.570715269 & 0.965827413 \\
\hline $\mathrm{N}$ & 1.245963989 & -0.275252548 & 0.887929947 \\
\hline $\mathrm{C}$ & 1.850567773 & 0.748966065 & 1.626450878 \\
\hline $\mathrm{C}$ & 2.443741762 & 0.547013073 & 2.895131895 \\
\hline
\end{tabular}

H $\quad 2.454575693 \quad-0.451905760 \quad 3.318725071$

C $\quad 2.997528341 \quad 1.598884614 \quad 3.611919562$

H $3.431645527 \quad 1.396052657 \quad 4.589396672$

C $\quad 2.996873776 \quad 2.913914284 \quad 3.121381401$

C $3.603426276 \quad 4.046228290 \quad 3.917560943$

H $3.444632631 \quad 5.010668533 \quad 3.425503543$

H $\quad 4.685300245 \quad 3.916268449 \quad 4.046934051$

H $3.167936306 \quad 4.111502794 \quad 4.922103772$

C $\quad 2.406284458 \quad 3.124490519 \quad 1.873761630$

H $\quad 2.384466357 \quad 4.136240068 \quad 1.475682364$

C $\quad \begin{array}{llll}1.839614572 & 2.080184859 & 1.129390119\end{array}$

$\begin{array}{lllll}\text { P } & 1.017783790 & 2.290418399 & -0.490823546\end{array}$

C $\quad-1.558585113-2.538312551 \quad 2.449340189$

$\begin{array}{lllll}\mathrm{H} & -2.579838909 & -2.143434289 & 2.385533167\end{array}$

C $-0.764018366-1.663726272 \quad 3.434418700$

H $\quad-1.263644995-1.654396715 \quad 4.410079640$

H $\quad-0.662520648 \quad-0.630022306 \quad 3.095539227$

H $\quad 0.244678241 \quad-2.063681913 \quad 3.578897198$

C $\quad-1.618296390 \quad-3.987172994 \quad 2.956456513$

H $\quad-0.623903327 \quad-4.446788294 \quad 2.957037933$

H $\quad-2.288567204-4.618176792 \quad 2.366013830$

H $\quad-1.984675007 \quad-3.999744920 \quad 3.990127425$

C $-1.610546379-3.788762843 \quad-0.254862605$

H $-1.407723118-4.686221116 \quad 0.342122081$

C $-3.134071877-3.615487132-0.370426250$

H $\quad-3.382316043-2.712781828-0.937942279$

H $\quad-3.627897676 \quad-3.5486191090 .604099376$

H $\quad-3.565799297-4.472856251 \quad-0.899556659$

C $\quad-0.970950811-3.970982821 \quad-1.636190643$

H $\quad-1.420133673-4.837528936-2.136463968$

H $\quad 0.107758322-4.134475675-1.573582055$

H $-1.140658381 \quad-3.088994157 \quad-2.256342441$

C $\quad 2.413064496 \quad 2.326819881 \quad-1.764882094$

H $\quad 1.884881341 \quad 2.409817568 \quad-2.721935504$

C $\quad 3.183805585 \quad 0.997444328 \quad-1.755928539$

$\begin{array}{lllll}\text { H } & 3.907460482 & 0.986338463 & -2.579904851\end{array}$

H $\quad 2.513532705 \quad 0.141867052 \quad-1.867195633$

$\begin{array}{lllll}\mathrm{H} & 3.742252823 & 0.876240791 & -0.821662027\end{array}$

C $3.370776514 \quad 3.518001424-1.609812777$

H $3.853966662 \quad 3.512606760 \quad-0.626870715$

H $2.875745766 \quad 4.484651294-1.743356431$

H $\quad 4.163005562 \quad 3.452057951 \quad-2.365185050$

$\begin{array}{lllll}\text { C } & 0.342631999 & 4.046837929 & -0.502737985\end{array}$

H $\quad 1.197585218 \quad 4.709612065 \quad-0.320737237$

$\begin{array}{lllll}\text { C } & -0.686345180 & 4.273955221 & 0.614044894\end{array}$

$\mathrm{H} \quad-1.010414632 \quad 5.321494240 \quad 0.611781219$

H $\quad-0.274817222 \quad 4.051243691 \quad 1.602146583$

H $\quad-1.574219961 \quad 3.653230023 \quad 0.467343276$

C $\quad-0.247284993 \quad 4.388322598 \quad-1.881670677$

H $\quad-1.078884705 \quad 3.722055906-2.134410568$

H $\quad 0.494093388 \quad 4.318698126-2.683212561$

H $\quad-0.632252015 \quad 5.414780265-1.876781688$

C $\quad-2.638599970 \quad 1.046658203 \quad-0.462046614$

$\begin{array}{llll}\mathrm{Si} & -3.817250162 & 1.324017222 & 0.975729741\end{array}$

C $\quad-2.949907172 \quad 1.664256801 \quad 2.636371769$

H $\quad-3.685224319 \quad 1.878842811 \quad 3.421609502$

$\mathrm{H} \quad-2.275281444 \quad 2.525811438 \quad 2.579365645$

H $\quad-2.356908935 \quad 0.806552698 \quad 2.971837436$

$\begin{array}{lllll}\text { C } & -4.963231097 & 2.810404209 & 0.648339327\end{array}$

H $\quad-5.528169232 \quad 2.676686943 \quad-0.281418343$

$\begin{array}{lllll}\mathrm{H} & -4.394590513 & 3.743467787 & 0.553448238\end{array}$

H $\quad-5.687245131 \quad 2.949396523 \quad 1.460811552$ 


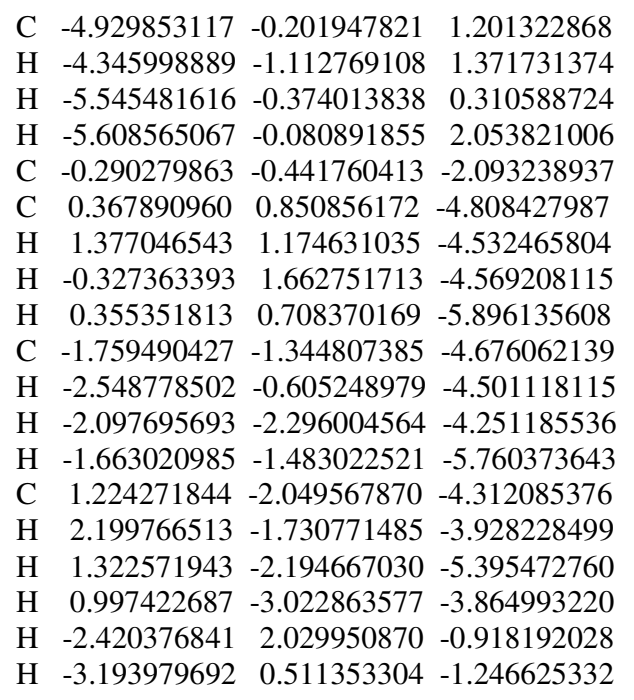

$==$
81

\begin{tabular}{|c|c|c|c|}
\hline $\mathrm{Nb}$ & -0.498088167 & 0.041176044 & +-0.787454275 \\
\hline $\mathrm{P}$ & -0.824486791 & -2.357762544 & 0.330020573 \\
\hline $\mathrm{C}$ & & -2.828212246 & 0.381274207 \\
\hline $\mathrm{C}$ & 0.011444500 & -0.861182305 & -4.008882440 \\
\hline $\mathrm{C}$ & 1.427713228 & -4.142591930 & 0.389067763 \\
\hline $\mathrm{H}$ & 0.721367139 & -4.969783436 & 0.392594227 \\
\hline $\mathrm{C}$ & 2.794414325 & -4.432785801 & 9849 \\
\hline $\mathrm{C}$ & 3.304580006 & -5.855388353 & 0.362521894 \\
\hline $\mathrm{H}$ & 2.486 & -6.57 & 690 \\
\hline $\mathrm{H}$ & & -6.03 & 96 \\
\hline $\mathrm{H}$ & & -6.0 & -0 . \\
\hline $\mathrm{C}$ & & -3.3 & 966 \\
\hline $\mathrm{H}$ & & -3.5 & \\
\hline $\mathrm{C}$ & 3.22 & -2.0 & \\
\hline $\mathrm{H}$ & & -1.2 & \\
\hline $\mathrm{C}$ & & -1.7 & \\
\hline $\mathrm{N}$ & & -0.4 & \\
\hline $\mathrm{C}$ & & 0.5 & \\
\hline $\mathrm{C}$ & & & \\
\hline $\mathrm{H}$ & & & \\
\hline $\mathrm{C}$ & & & \\
\hline $\mathrm{H}$ & & & \\
\hline $\mathrm{C}$ & & 2.6 & \\
\hline $\mathrm{C}$ & & & \\
\hline $\mathrm{H}$ & & 4.7 & \\
\hline $\mathrm{H}$ & & & \\
\hline $\mathrm{H}$ & 3.7 & 3.7 & \\
\hline $\mathrm{C}$ & 2.6 & & \\
\hline $\mathrm{H}$ & 2.5 & 3.9 & \\
\hline $\mathrm{C}$ & 1.9 & 1.9 & \\
\hline $\mathrm{P}$ & 33 & 2.2 & -0. \\
\hline $\mathrm{C}$ & -1.3 & -2.4 & \\
\hline $\mathrm{H}$ & -2.3 & -1.99 & \\
\hline $\mathrm{C}$ & -0.466309884 & -1.515 & \\
\hline $\mathrm{H}$ & -0.875876585 & -1.4 & \\
\hline $\mathrm{H}$ & & -0.504935796 & \\
\hline $\mathrm{H}$ & & & \\
\hline $\mathrm{C}$ & & & \\
\hline $\mathrm{H}$ & & & \\
\hline $\mathrm{H}$ & -2.162157642 & -4.472606283 & 2.272842364 \\
\hline
\end{tabular}

$\begin{array}{llll}\text { H } & -1.676011944 & -3.773464770 & 3.816836679\end{array}$

$\begin{array}{lllll}\text { C } & -1.747308669 & -3.817926869 & -0.417425428\end{array}$

$\begin{array}{lllll}\text { H } & -1.527187730 & -4.688205954 & 0.212215612\end{array}$

$\begin{array}{llll}\text { C } & -3.262644329 & -3.558529857 & -0.380886218\end{array}$

$\begin{array}{lllll}\mathrm{H} & -3.519741597 & -2.666037897 & -0.960009464\end{array}$

$\begin{array}{lllll}\mathrm{H} & -3.642734140 & -3.420462793 & 0.635866936\end{array}$

$\begin{array}{lllll}\mathrm{H} & -3.795797910 & -4.410276994 & -0.819028334\end{array}$

$\begin{array}{lllll}\text { C } & -1.286108076 & -4.123484347 & -1.845272468\end{array}$

H $\quad-1.859464040 \quad-4.966522676 \quad-2.249338974$

$\begin{array}{lllll}\mathrm{H} & -0.225657884 & -4.384930434 & -1.889432403\end{array}$

$\begin{array}{lllll}\text { H } & -1.449403592 & -3.257529869 & -2.490006393\end{array}$

C $\quad 2.339823938 \quad 2.497937532-2.195170381$

$\begin{array}{llll}\text { H } & 1.766998360 & 2.510879466 & -3.129497332\end{array}$

$\begin{array}{lllll}\text { C } & 3.282919210 & 1.285370957 & -2.214159814\end{array}$

H $3.934198255 \quad 1.328076624-3.095118957$

$\begin{array}{lllll}\mathrm{H} & 2.739304750 & 0.339422091 & -2.238825912\end{array}$

$\begin{array}{lllll}\mathrm{H} & 3.921588801 & 1.279986269 & -1.325024722\end{array}$

C $3.156266514 \quad 3.796101460-2.100472515$

H $3.673630790 \quad 3.873299186-1.138116220$

H $\quad 2.548850543 \quad 4.694685765 \quad-2.239189141$

$\begin{array}{lllll}\mathrm{H} & 3.924746268 & 3.800774619 & -2.883317941\end{array}$

$\begin{array}{lllll}\text { C } & 0.315389783 & 4.004940167 & -0.654846568\end{array}$

$\begin{array}{lllll}\mathrm{H} & 1.171493623 & 4.687874555 & -0.614065447\end{array}$

$\begin{array}{llll}\text { C } & -0.485087010 & 4.166388614 & 0.641157977\end{array}$

$\begin{array}{lllll}\mathrm{H} & -0.900800989 & 5.179658545 & 0.698984082\end{array}$

$\begin{array}{lllll}\mathrm{H} & 0.130882196 & 4.002676594 & 1.529122553\end{array}$

$\begin{array}{lllll}\mathrm{H} & -1.313259696 & 3.457186701 & 0.668908589\end{array}$

C $\quad-0.535574502 \quad 4.360094119-1.885518393$

$\begin{array}{lllll}\mathrm{H} & -1.390074210 & 3.681269934 & -1.973699777\end{array}$

$\begin{array}{lllll}\mathrm{H} & 0.035803142 & 4.306147814 & -2.818303434\end{array}$

$\begin{array}{lllll}\mathrm{H} & -0.922688080 & 5.381699509 & -1.793136075\end{array}$

$\begin{array}{lllll}\text { C } & -2.264402477 & 0.751301994 & -0.243126039\end{array}$

$\begin{array}{llll}\text { C } & -3.367722438 & 1.181221118 & 0.715886776\end{array}$

$\begin{array}{llll}\text { C } & -2.855808206 & 1.368348569 & 2.157680071\end{array}$

$\begin{array}{llll}\mathrm{H} & -3.668451980 & 1.689054829 & 2.821327188\end{array}$

$\begin{array}{llll}\mathrm{H} & -2.062645750 & 2.119646476 & 2.212031535\end{array}$

$\begin{array}{lllll}\mathrm{H} & -2.453960283 & 0.431959045 & 2.554463623\end{array}$

$\begin{array}{lllll}\text { C } & -4.027474238 & 2.495192513 & 0.226675089\end{array}$

$\begin{array}{lllll}\mathrm{H} & -4.436235744 & 2.373105635 & -0.782983590\end{array}$

$\begin{array}{lllll}\mathrm{H} & -3.315328989 & 3.323907517 & 0.198886178\end{array}$

$\begin{array}{llll}\mathrm{H} & -4.852736186 & 2.784561172 & 0.889864559\end{array}$

$\begin{array}{llll}\text { C } & -4.470636714 & 0.091334145 & 0.725912803\end{array}$

$\begin{array}{lllll}\mathrm{H} & -4.076935020 & -0.865803502 & 1.079450029\end{array}$

$\begin{array}{lllll}\mathrm{H} & -4.878769715 & -0.066339460 & -0.278707621\end{array}$

$\begin{array}{llll}\mathrm{H} & -5.299191032 & 0.383279195 & 1.384655169\end{array}$

$\begin{array}{lllll}\text { C } & -0.654012078 & -0.333525461 & -2.736808639\end{array}$

$\begin{array}{lllll}\text { C } & 0.540137194 & 0.335138268 & -4.840097461\end{array}$

$\begin{array}{lllll}\mathrm{H} & 1.353920648 & 0.845531641 & -4.320682564\end{array}$

$\begin{array}{lllll}\mathrm{H} & -0.254741379 & 1.065485667 & -5.028530562\end{array}$

H $\quad 0.920874145 \quad-0.007217498 \quad-5.811414328$

$\begin{array}{llll}\text { C } & -1.030458699 & -1.595763843 & -4.888423898\end{array}$

$\begin{array}{lllll}\mathrm{H} & -1.466981987 & -2.455372878 & -4.372789777\end{array}$

$\begin{array}{lllll}\mathrm{H} & -0.568032491 & -1.961246382 & -5.814061282\end{array}$

$\begin{array}{lllll}\mathrm{H} & -1.848970481 & -0.921710110 & -5.166914145\end{array}$

$\begin{array}{llll}\text { C } & 1.181804527 & -1.819018905 & -3.710047684\end{array}$

H $\quad 0.837249593 \quad-2.715118126-3.187822235$

H $\quad 1.939051450 \quad-1.342476199 \quad-3.081007590$

H $1.672745773 \quad-2.137009760-4.638586650$

$\begin{array}{lllll}\mathrm{H} & -2.681064417 & 0.704971102 & -1.269725834\end{array}$

H $\quad-1.6451503650 .075735682 \quad-2.994531440$

80 


\begin{tabular}{|c|c|c|c|}
\hline $\mathrm{Nb}$ & -0.618049324 & $4-0.037401835$ & -0.735023190 \\
\hline $\mathrm{P}$ & -0.865788380 & -2.448011966 & 0.346834135 \\
\hline $\mathrm{C}$ & 0.891989448 & -2.773998292 & 0.730237759 \\
\hline $\mathrm{C}$ & -0.999732361 & -0.411620085 & -4.070239430 \\
\hline $\mathrm{C}$ & 1.439538303 & -4.052103917 & 0.917292681 \\
\hline $\mathrm{H}$ & 0.786636602 & -4.920818580 & 0.871544968 \\
\hline $\mathrm{C}$ & 2.801769256 & -4.255999444 & 1.142942453 \\
\hline $\mathrm{C}$ & 3.383688190 & -5.638450627 & 1.329486705 \\
\hline $\mathrm{H}$ & 2.604532479 & -6.406568166 & 1.307002197 \\
\hline $\mathrm{H}$ & 3.910914652 & -5.729647654 & 2.287220162 \\
\hline $\mathrm{H}$ & 4.108983885 & -5.881313340 & 0.542539206 \\
\hline $\mathrm{C}$ & 3.618773424 & -3.114745293 & 1.163713698 \\
\hline $\mathrm{H}$ & 4.691959449 & -3.229613097 & 1.307058228 \\
\hline $\mathrm{C}$ & 3.099849952 & -1.838987593 & 0.993717594 \\
\hline $\mathrm{H}$ & 3.770732633 & -0.986635089 & 1.011866810 \\
\hline $\mathrm{C}$ & 1.715322087 & -1.620669742 & 176 \\
\hline $\mathrm{N}$ & 1.166274503 & -0.338881250 & 0.604463377 \\
\hline $\mathrm{C}$ & 1.757725319 & 0.740506171 & 1.286970756 \\
\hline $\mathrm{C}$ & 2.340133438 & 0.608901645 & 2.570630891 \\
\hline $\mathrm{H}$ & 2.346829908 & -0.364385026 & 3.049618876 \\
\hline $\mathrm{C}$ & 2.89 & 1.694 & 118 \\
\hline $\mathrm{H}$ & 3.324573068 & 1.541912237 & 4.221949015 \\
\hline $\mathrm{C}$ & 2.908060681 & 2.980013003 & 2.669 \\
\hline $\mathrm{C}$ & 3.525971028 & 4.149843959 & 3.400517636 \\
\hline $\mathrm{H}$ & 3.361357079 & 5.088922264 & 2.8631 \\
\hline $\mathrm{H}$ & 4.609629723 & 1818 & 532 \\
\hline $\mathrm{H}$ & 3.104277368 & 4.265734034 & 4.4065 \\
\hline $\mathrm{C}$ & 2.310168030 & 3.124692617 & 1.416636 \\
\hline $\mathrm{H}$ & 2.282580243 & 4.114993852 & 0.967606567 \\
\hline $\mathrm{C}$ & 1.733091186 & 2.045 & 0.7 \\
\hline $\mathrm{P}$ & 0.859499448 & 2.183045343 & -0.8679 \\
\hline $\mathrm{C}$ & -1.751887620 & -2.572215781 & 2.0188 \\
\hline $\mathrm{H}$ & -2.783475224 & -2.274436698 & 56132 \\
\hline $\mathrm{C}$ & -1.159741184 & -1.550776261 & 3.00 \\
\hline $\mathrm{H}$ & -1.75 & -1.53518 & 3.9 \\
\hline $\mathrm{H}$ & & -0.538843202 & 3858 \\
\hline $\mathrm{H}$ & -0.132061731 & -1.817583325 & 3.270 \\
\hline $\mathrm{C}$ & -1.761554051 & -3.976141742 & 2.641823838 \\
\hline $\mathrm{H}$ & -0.742765363 & -4.343396958 & 2.80 \\
\hline $\mathrm{H}$ & -2.295407787 & -4.708795396 & 2.029490808 \\
\hline $\mathrm{H}$ & -2.25953036 & -3.944223499 & 3.618697380 \\
\hline $\mathrm{C}$ & -1.457748993 & -3.960994880 & -0.602372106 \\
\hline $\mathrm{H}$ & -1.356305658 & -4.819431273 & 0.072582802 \\
\hline $\mathrm{C}$ & -2.942136161 & -3.803952946 & -0.9704 \\
\hline $\mathrm{H}$ & -3.09252 & -2.934884563 & -1.619074007 \\
\hline $\mathrm{H}$ & -3.580298510 & -3.679959239 & -0.089369856 \\
\hline $\mathrm{H}$ & -3.290584655 & -4.693081665 & -1.509098418 \\
\hline $\mathrm{C}$ & -0.597983274 & -4.221293751 & -1.846581039 \\
\hline $\mathrm{H}$ & -1.006248439 & -5.070094778 & -2.408381245 \\
\hline $\mathrm{H}$ & 0.437643646 & -4.455233804 & -1.587331996 \\
\hline $\mathrm{H}$ & -0.591178880 & -3.348417486 & -2.503285772 \\
\hline $\mathrm{C}$ & 2.247762021 & 2.231673012 & -2.165126890 \\
\hline $\mathrm{H}$ & 1.714601138 & 2.201759225 & -3.122509889 \\
\hline $\mathrm{C}$ & 3.124376616 & 0.972840255 & -2.064576993 \\
\hline $\mathrm{H}$ & 3.850649112 & 0.960850988 & -2.886065820 \\
\hline $\mathrm{H}$ & 2.538845736 & 0.052394247 & -2.117959775 \\
\hline $\mathrm{H}$ & 3.684647161 & 0.960811419 & -1.123979364 \\
\hline $\mathrm{C}$ & 3.124913165 & 3.492657233 & -2.116346122 \\
\hline $\mathrm{H}$ & 3.611153250 & 3.601018985 & -1.140661169 \\
\hline $\mathrm{H}$ & 2.566137362 & 4.409293867 & -2.325256533 \\
\hline $\mathrm{H}$ & 3.918770346 & 3.418118516 & -2.869633506 \\
\hline
\end{tabular}

C $\quad 0.155489907 \quad 3.926237301 \quad-0.900254934$

H $\quad 1.002841675 \quad 4.616079901 \quad-0.809643646$

C $\quad-0.794818993 \quad 4.170349003 \quad 0.278794006$

H $\quad-1.211465719 \quad 5.182896237 \quad 0.218671727$

$\begin{array}{llll}\mathrm{H} & -0.288260587 & 4.069622215 & 1.242232169\end{array}$

$\begin{array}{llll}\mathrm{H} & -1.623468895 & 3.458928045 & 0.257165593\end{array}$

C $\quad-0.545157500 \quad 4.188556985 \quad-2.242635984$

H $\quad-1.380978528 \quad 3.496087040 \quad-2.386132064$

H $\quad 0.132959395 \quad 4.082358239-3.095583477$

H $\quad-0.945004533 \quad 5.209064635 \quad-2.264148360$

C $\quad-2.417467674 \quad 0.605087313 \quad-0.381519739$

$\begin{array}{llll}\text { C } & -3.712106941 & 1.072860286 & 0.254389196\end{array}$

$\begin{array}{llll}\text { C } & -3.485637696 & 1.470994037 & 1.727643617\end{array}$

H $\quad-2.750856868 \quad 2.276684449 \quad 1.816195215$

$\mathrm{H} \quad-4.420793783 \quad 1.814300885 \quad 2.186375255$

H $\quad-3.118805463 \quad 0.620384540 \quad 2.309923703$

C $\quad-4.295415309 \quad 2.277500527 \quad-0.522889180$

H $\quad-5.243295653 \quad 2.604105482-0.077400291$

H $\quad-4.489265242 \quad 2.011757668$ - 1.567976089

H $\quad-3.609728796 \quad 3.129280149 \quad-0.516148591$

$\begin{array}{llll}\text { C } & -4.750397902 & -0.077468737 & 0.204475691\end{array}$

$\begin{array}{llll}\mathrm{H} & -5.701113594 & 0.236671617 & 0.653550614\end{array}$

H $\quad-4.390100213-0.954000351 \quad 0.751514718$

H $\quad-4.949285389-0.385083099 \quad-0.827776434$

C $-0.432128010 \quad-0.486476718 \quad-2.657000109$

C $\quad-0.228594043 \quad 0.683468425 \quad-4.851998380$

H $\quad 0.845809538 \quad 0.468013681 \quad-4.876281637$

H $\quad-0.369346632 \quad 1.663483610 \quad-4.385360867$

$\begin{array}{lllll}\mathrm{H} & -0.581103308 & 0.746100770 & -5.889840814\end{array}$

C $\quad-2.498793663-0.058133677 \quad-4.083368187$

H $\quad-2.6806052820 .914831809 \quad-3.614734572$

H $\quad-3.085643449-0.809397750 \quad-3.543157742$

H $\quad-2.877627983-0.011076930-5.111641785$

C $\quad-0.804353133 \quad-1.754928090 \quad-4.813838230$

H $\quad 0.247450098 \quad-2.063941840 \quad-4.806535342$

H $\quad-1.119937923-1.668652771 \quad-5.861275035$

H $\quad-1.394557144 \quad-2.552658987-4.353314475$

H $\quad-2.560098420 \quad 0.373910161 \quad-1.476637702$

H $\quad 0.608941880-0.876557285-2.677966618$

\begin{tabular}{|c|c|c|c|}
\hline \multicolumn{4}{|l|}{82} \\
\hline $\mathrm{Nb}$ & -0.583039426 & 0.128259172 & -0.619938268 \\
\hline $\mathrm{P}$ & -0.941996135 & -2.265185183 & 0.494662040 \\
\hline $\mathrm{C}$ & 0.799490501 & -2.718705997 & 0.813536333 \\
\hline $\mathrm{C}$ & -1.285684216 & -0.157251041 & -3.935781563 \\
\hline $\mathrm{C}$ & 1.254240449 & -4.033815618 & 0.999875600 \\
\hline $\mathrm{H}$ & 0.533073780 & -4.847745405 & 1.015552767 \\
\hline $\mathrm{C}$ & 2.606246298 & -4.343943442 & 1.147700712 \\
\hline $\mathrm{C}$ & 3.085340960 & -5.764142878 & 1.342334209 \\
\hline $\mathrm{H}$ & 2.248208083 & -6.468495413 & 1.368775525 \\
\hline $\mathrm{H}$ & 3.642922038 & -5.877857904 & 2.280404315 \\
\hline $\mathrm{H}$ & 3.756531839 & -6.078923290 & 0.533243003 \\
\hline $\mathrm{C}$ & 3.513036157 & -3.273671465 & 1.081015612 \\
\hline $\mathrm{H}$ & 4.580589297 & -3.473573414 & 1.157375669 \\
\hline $\mathrm{C}$ & 3.088803851 & -1.964772076 & 0.906985226 \\
\hline $\mathrm{H}$ & 3.825992094 & -1.170575358 & 0.855510353 \\
\hline $\mathrm{C}$ & 1.715071044 & -1.636137030 & 0.795705433 \\
\hline $\mathrm{N}$ & 1.261903957 & -0.318099288 & 0.615560460 \\
\hline $\mathrm{C}$ & 1.987217189 & 0.712313065 & 1.237421596 \\
\hline $\mathrm{C}$ & 2.637377411 & 0.546668871 & 2.485614603 \\
\hline $\mathrm{H}$ & 2.583086541 & -0.415096109 & 2.984395078 \\
\hline
\end{tabular}


C $\quad 3.335793526 \quad 1.583018637 \quad 3.086653472$

H $3.811450909 \quad 1.405952991 \quad 4.049923986$

C $3.431806017 \quad 2.853006607 \quad 2.495433308$

C $4.206413683 \quad 3.968244386 \quad 3.159036333$

H $4.084650952 \quad 4.914425047 \quad 2.622748575$

H $\quad 5.280898537 \quad 3.747898079 \quad 3.198360209$

H $\quad 3.876664429 \quad 4.128568877 \quad 4.192875891$

C $\quad 2.765203356 \quad 3.035398746 \quad 1.283780887$

$\begin{array}{llll}\mathrm{H} & 2.797070393 & 4.018733433 & 0.820381858\end{array}$

C $\quad 2.043341670 \quad 2.006005022 \quad 0.659179530$

$\begin{array}{lllll}\mathrm{P} & 1.054478667 & 2.217172855 & -0.863577535\end{array}$

C $-1.794730831 \quad-2.333431629 \quad 2.186783235$

H $\quad-2.811796075 \quad-1.975490891 \quad 1.982252539$

C $-1.124065970 \quad-1.348826817 \quad 3.157635807$

H $\quad-1.675793006-1.326445176 \quad 4.104746752$

H $-1.096775391 \quad-0.332145867 \quad 2.759315249$

H $\quad-0.095977739 \quad-1.655031833 \quad 3.377186254$

C $-1.871663421 \quad-3.734340759 \quad 2.812662583$

H $\quad-0.872027481 \quad-4.153934927 \quad 2.968550744$

H -2.449479068 -4.439741603 2.208284764

H $\quad-2.357239579 \quad-3.673575962 \quad 3.794270325$

C $-1.642733738-3.749046045-0.429343306$

$\begin{array}{llll}\text { H } & -1.526852695 & -4.612306223 & 0.237021405\end{array}$

C $-3.139631038-3.553531126-0.712357132$

$\mathrm{H} \quad-3.303339853-2.684086934-1.355622737$

$\mathrm{H} \quad-3.722952223 \quad-3.412460640 \quad 0.203633443$

H $-3.541107453-4.434547122-1.226663709$

C $-0.861209517-4.033028953-1.719780496$

H $\quad-1.306784145 \quad-4.888378559-2.242004672$

H $\quad 0.187321407-4.269037773-1.519990446$

$\begin{array}{lllll}\text { H } & -0.893855983 & -3.171088482 & -2.390788353\end{array}$

C $\quad 2.312552626 \quad 2.197672963-2.281854686$

H $\quad 1.6902240692 .245164178-3.184551476$

C $3.075787300 \quad 0.863779246-2.293248678$

$\mathrm{H} \quad 3.739088006 \quad 0.822077662-3.165193889$

H $\quad 2.401881105 \quad 0.005420170-2.340489348$

H $\quad 3.695716904 \quad 0.759894031 \quad-1.396640728$

C $3.295288883 \quad 3.378596707-2.286647908$

H $3.888594101 \quad 3.404303993-1.366318051$

H $\quad 2.797992972 \quad 4.346192980-2.401406211$

H $3.995555212 \quad 3.272063146-3.124128653$

C $\quad 0.475911383 \quad 4.007875474-0.796881115$

H $\quad 1.381748456 \quad 4.625565968$-0.771914429

C $\quad-0.333699910 \quad 4.295275336 \quad 0.475105154$

H $\quad-0.672936428 \quad 5.338199755 \quad 0.470571486$

H $\quad 0.257393807 \quad 4.137027990 \quad 1.380903700$

H $\quad-1.215109132 \quad 3.651571992 \quad 0.527880950$

C $\quad-0.319024214 \quad 4.366512207-2.061207224$

$\mathrm{H} \quad-1.222251144 \quad 3.754506160-2.139224819$

H $\quad 0.266053106 \quad 4.226073963-2.976140507$

$\mathrm{H} \quad-0.626122318 \quad 5.418265151-2.024409689$

C $\quad-2.190872138 \quad 0.975973296 \quad 0.152420311$

C $\quad-3.675233675 \quad 1.329632242 \quad 0.135974602$

C $-4.247045892 \quad 1.257840652 \quad 1.571782603$

$\mathrm{H} \quad-5.319660186 \quad 1.488801500 \quad 1.580933700$

$\begin{array}{llll}\mathrm{H} & -3.743822959 & 1.972442550 & 2.233553816\end{array}$

$\begin{array}{llll}\mathrm{H} & -4.115385427 & 0.256739687 & 1.997235930\end{array}$

C $\quad-3.895669615 \quad 2.762379867 \quad-0.406534371$

H $\quad-3.504932028 \quad 2.858880085-1.423867692$

$\begin{array}{lllll}\mathrm{H} & -3.395976208 & 3.507990215 & 0.220184965\end{array}$

H $\quad-4.965654467 \quad 3.007146856 \quad-0.425647782$

$\begin{array}{llll}\text { C } & -4.460451219 & 0.339684879 & -0.749295791\end{array}$
H $\quad-4.379111032-0.678600145 \quad-0.357066875$

H $\quad-4.074919197 \quad 0.333233198 \quad-1.772028043$

$\mathrm{H} \quad-5.523904774 \quad 0.608163723 \quad-0.786722030$

C $\quad-0.685563572-0.309498612-2.541918257$

C $\quad-2.087049902 \quad 1.156806955-4.046051357$

H $\quad-1.437289945 \quad 2.021099708$-3.878110539

H $\quad-2.889420705 \quad 1.195278787 \quad-3.304754157$

H $\quad-2.537868153 \quad 1.255907571 \quad-5.041692471$

C $-2.226294473-1.341856549-4.262513671$

H $\quad-1.687646147 \quad-2.294993490 \quad-4.247012221$

H $\quad-2.663534651-1.222977545 \quad-5.262360205$

H $\quad-3.043298235-1.403094710 \quad-3.537391374$

C $-0.155020203-0.126022073-4.991480907$

H $\quad 0.424863386-1.056301485-4.978485084$

H $\quad 0.536202021 \quad 0.702536635-4.801767031$

H $\quad-0.564011651 \quad 0.001479715 \quad-6.001485098$

$\mathrm{H} \quad-1.724549231 \quad 1.255021970 \quad 1.128461404$

H $\quad 0.245347656 \quad-0.926901630 \quad-2.572423671$

87

$\mathrm{Nb} \quad-0.751703264 \quad-0.033417653 \quad-0.414303992$

$\begin{array}{llll}\mathrm{P} & -1.079705326 & -2.370018722 & 0.873201591\end{array}$

C $\quad 0.659421100 \quad-2.739475006 \quad 1.286229926$

$\begin{array}{llll}\text { C } & -1.725563444 & -0.250095795 & -3.776830449\end{array}$

$\begin{array}{lrrrr}\text { C } & 1.176547384 & -4.029557479 & 1.476032767\end{array}$

$\begin{array}{llll}\mathrm{H} & 0.507736014 & -4.885494675 & 1.422943314\end{array}$

$\begin{array}{llll}\text { C } & 2.531693122 & -4.261619326 & 1.717227585\end{array}$

$\begin{array}{llll}\text { C } & 3.082488643 & -5.655993656 & 1.908692737\end{array}$

$\begin{array}{llll}\text { H } & 2.287062136 & -6.407234767 & 1.880457360\end{array}$

$\begin{array}{lllll}\mathrm{H} & 3.599886584 & -5.758538090 & 2.870655377\end{array}$

$\begin{array}{llll}\mathrm{H} & 3.808479223 & -5.915393188 & 1.127577427\end{array}$

$\begin{array}{llll}\text { C } & 3.370824815 & -3.136243278 & 1.750473612\end{array}$

$\begin{array}{llll}\text { H } & 4.439438166 & -3.273756157 & 1.908019400\end{array}$

C $\quad 2.882322960-1.849585063 \quad 1.573950481$

$\begin{array}{lllll}\mathrm{H} & 3.572076334 & -1.012438823 & 1.598756893\end{array}$

$\begin{array}{llll}\text { C } & 1.504730429 & -1.599105622 & 1.354121732\end{array}$

$\begin{array}{lllll}\mathrm{N} & 0.978166698 & -0.317656082 & 1.147008717\end{array}$

$\begin{array}{lllll}\text { C } & 1.565498337 & 0.758170694 & 1.815791938\end{array}$

$\begin{array}{lllll}\text { C } & 2.140170436 & 0.645701097 & 3.107661436\end{array}$

$\begin{array}{llll}\text { H } & 2.135571289 & -0.320342135 & 3.601012519\end{array}$

$\begin{array}{llll}\text { C } & 2.692660825 & 1.736345808 & 3.760530106\end{array}$

$\begin{array}{lllll}\text { H } & 3.109468407 & 1.595631735 & 4.756519362\end{array}$

$\begin{array}{llll}\text { C } & 2.714633463 & 3.015821754 & 3.181057817\end{array}$

$\begin{array}{llll}\text { C } & 3.332878115 & 4.191527256 & 3.901593748\end{array}$

H $\quad 3.176513983 \quad 5.1248622603 .351800874$

H $\quad 4.415124650 \quad 4.063743348 \quad 4.032399106$

$\begin{array}{lllll}\mathrm{H} & 2.904500852 & 4.321545981 & 4.903075593\end{array}$

$\begin{array}{llll}\text { C } & 2.129045119 & 3.145827655 & 1.922259284\end{array}$

$\begin{array}{llll}\mathrm{H} & 2.111283505 & 4.130680627 & 1.461486367\end{array}$

$\begin{array}{llll}\text { C } & 1.555992072 & 2.058575358 & 1.242925022\end{array}$

$\begin{array}{lllll}\text { P } & 0.733889230 & 2.214411219 & -0.377893723\end{array}$

C $\quad-1.995167338 \quad-2.371860317 \quad 2.528711163$

$\begin{array}{llll}\text { H } & -3.016859948 & -2.069852535 & 2.269315725\end{array}$

C $-1.398665714-1.308996101 \quad 3.464944240$

$\begin{array}{llll}\text { H } & -2.006084032 & -1.236286652 & 4.375047659\end{array}$

H $\quad-1.361456156 \quad-0.325132464 \quad 2.993046495$

H $\quad-0.380845180 \quad-1.579537186 \quad 3.764117829$

$\begin{array}{lllll}\text { C } & -2.032032227 & -3.743986235 & 3.219318881\end{array}$

H $\quad-1.020203842-4.115691570 \quad 3.413924791$

H $\quad-2.569593380-4.499577587 \quad 2.638877035$

H $\quad-2.540150904 \quad-3.6551115694 .187117901$ 
C $-1.722590370 \quad-3.899203908-0.014522029$

$\begin{array}{llll}\text { H } & -1.571755914 & -4.733999623 & 0.679846824\end{array}$

C $\quad-3.227170641-3.772604353-0.304254978$

H $-3.434163956-2.918008913-0.955598562$

$\begin{array}{lllll}\mathrm{H} & -3.820265134 & -3.651665675 & 0.607186485\end{array}$

H $-3.584105262-4.676052122-0.812310026$

C $-0.936653422-4.201325076-1.298835288$

$\mathrm{H} \quad-1.272280362-5.157078554-1.718495951$

H $\quad 0.139042851 \quad-4.273134345-1.118344286$

H $-1.105586118-3.432454963-2.058098984$

C $2.134077058 \quad 2.226536849-1.660014766$

H $\quad 1.6118946492 .239776612-2.626081637$

C $\quad 2.949756555 \quad 0.926699112-1.569279177$

H $\quad 3.706568659 \quad 0.907036785-2.362041843$

H $\quad 2.328616590 \quad 0.033842527 \quad-1.677910964$

H $\quad 3.469127847 \quad 0.857443226 \quad-0.608394789$

C $3.061782255 \quad 3.448324940-1.579359144$

H $3.545654782 \quad 3.512985844 \quad-0.598965520$

H $\quad 2.540019326 \quad 4.391053809 \quad-1.767896041$

H $3.854638253 \quad 3.359332321 \quad-2.331974713$

C $\quad 0.056900433 \quad 3.966724148 \quad-0.436292386$

H $\quad 0.917767464 \quad 4.635107977-0.314119615$

C $\quad-0.924198199 \quad 4.229826338 \quad 0.713113722$

$\mathrm{H} \quad-1.308229114 \quad 5.254559790 \quad 0.645173935$

$\begin{array}{lllll}\mathrm{H} & -0.453025446 & 4.108179907 & 1.691679240\end{array}$

$\begin{array}{lllll}\mathrm{H} & -1.771959108 & 3.543904294 & 0.660857934\end{array}$

C $\quad-0.588343207 \quad 4.253544047-1.801162343$

H $\quad-1.434228102 \quad 3.584721092-1.983937044$

H $\quad 0.116866613 \quad 4.138954624 \quad-2.630866828$

H $\quad-0.961359558 \quad 5.284071512-1.826589363$

$\begin{array}{llll}\text { C } & -2.388400092 & 0.633482768 & 0.035025893\end{array}$

C $\quad-3.753993426 \quad 1.102290734 \quad 0.482767655$

C $\quad-3.739500936 \quad 1.591020117 \quad 1.951019305$

$\begin{array}{llll}\mathrm{H} & -4.736185759 & 1.933315361 & 2.258193419\end{array}$

H $\quad-3.039555294 \quad 2.419672824 \quad 2.089722637$

$\begin{array}{llll}\mathrm{H} & -3.436469782 & 0.785604154 & 2.626125214\end{array}$

C $-4.258480426 \quad 2.242821602-0.432728137$

H $\quad-5.258296373 \quad 2.570224201-0.120234336$

H $\quad-3.595744488 \quad 3.111941611-0.395086433$

$\begin{array}{lllll}\text { H } & -4.317652237 & 1.909342681 & -1.472744423\end{array}$

$\begin{array}{llll}\text { C } & -4.753828365 & -0.074791929 & 0.370412086\end{array}$

$\begin{array}{llll}\mathrm{H} & -5.756785056 & 0.244871513 & 0.680429286\end{array}$

H $-4.815065386-0.442985224-0.657563464$

H $\quad-4.454245845 \quad-0.908988725 \quad 1.011596534$

$\begin{array}{llll}\text { C } & -0.740818026 & -0.418851480 & -2.598207203\end{array}$

$\begin{array}{lllll}\text { C } & -2.098360904 & 1.233652614 & -3.943984562\end{array}$

H $\quad-1.207953580 \quad 1.842865944 \quad-4.143135125$

H $\quad-2.792227559 \quad 1.378663104 \quad-4.781576810$

H $\quad-2.570683070 \quad 1.612345466 \quad-3.033520192$

C $-3.006559628-1.064669132-3.523656866$

H $\quad-3.724465844-0.943170576-4.344210301$

H $-2.782774796 \quad-2.135362589-3.438331209$

H $\quad-3.481443108-0.742577914 \quad-2.592604868$

C $\quad-1.072431791-0.745879375 \quad-5.088795473$

H $\quad-0.160974575-0.178681764-5.313068604$

Н $-1.751233329-0.638101306-5.944664809$

H $\quad-0.794324478-1.804106982-5.015707148$

$\mathrm{H} \quad 0.195213276 \quad 0.122125662-2.853113910$

$\mathrm{H} \quad-0.411413570 \quad-1.481135813-2.561407590$ 


\section{S8. Computed components}

\begin{tabular}{|l|l|l|}
\hline & $\mathrm{E}(\mathrm{SCF})(\mathrm{eV}, \mathrm{LACVP} * *)$ & $\mathrm{E}(\mathrm{SCF})(\mathrm{eV}, \mathrm{cc}-\mathrm{pVTZ}(-\mathrm{f}))$ \\
\hline $\mathbf{7 1}$ & -73598.687 & -73612.943 \\
\hline $\mathbf{7 3}$ & -73598.842 & -73613.103 \\
\hline $\mathbf{7 2}$ & -73598.672 & -73612.946 \\
\hline $\mathbf{7 7}$ & -73598.232 & -73612.510 \\
\hline $\mathbf{6 4}$ & -73598.271 & -73598.271 \\
\hline $\mathbf{8 1}$ & -59914.972 & -59928.023 \\
\hline $\mathbf{8 0}$ & -59915.233 & -59928.280 \\
\hline $\mathbf{8 2}$ & -59914.900 & -59927.966 \\
\hline $\mathbf{8 7}$ & -59914.684 & -59927.743 \\
\hline
\end{tabular}

\section{References}

(1) Pangborn, A. B.; Giardello, M. A.; Grubbs, R. H.; Rosen, R. K.; Timmers, F. J. Organometallics 1996, 15, 1518-1520.

(2) Kilgore, U. J.; Yang, X.; Tomaszewski, J.; Huffman, J. C.; Mindiola, D. J. Inorg. Chem. 2006, 45, 10712-10721.

(3) Tang, H.; Richey, H. G. Organometallics 2001, 20, 1569-1574.

(4) Schrock, R. R.; Fellmann, J. D. J. Am. Chem Soc. 1978, 100, 3359-70.

(5) Jaguar 5.5 ed, Schrödinger, L.L.C, Portland, OR, 1991-2003.

(6) Becke, A. D. Phys. Rev. A 1988, 38, 3098.

(7) Becke, A. D. J. Chem. Phys. 1993, 98, 5648.

(8) Lee, C. T.; Yang, W. T.; Parr, R. G. Phys. Rev. B 1988, 37, 785.

(9) Vosko, S. H.; Wilk, L.; Nusair, M. Can. J. Phys. 1980, 58, 1200.

(10) Hay, P. J.; Wadt, W. R. J. Chem. Phys. 1985, 82, 270.

(11) Hay, P. J.; Wadt, W. R. J. Chem. Phys. 1985, 82, 299.

(12) Wadt, W. R.; Hay, P. J. J. Chem. Phys. 1985, 82, 284.

(13) Dunning, T. H. J. Chem. Phys. 1989, 90, 1007. 